\title{
Low-level blast exposure induces chronic vascular remodeling, perivascular astrocytic degeneration and vascular-associated neuroinflammation
}

Miguel A. Gama Sosa 1,2,3* , Rita De Gasperi' ${ }^{2,3,4}$, Dylan Pryor ${ }^{4}$, Georgina S. Perez Garcia ${ }^{3,4,5}$, Gissel M. Perez ${ }^{4}$, Rania Abutarboush 7,8 , Usmah Kawoos ${ }^{7,8}$, Seth Hogg ${ }^{9}$, Benjamin Ache9 ${ }^{9}$, William G. Janssen ${ }^{3,6}$, Allison Sowa ${ }^{3,6}$, Timothy Tetreault ${ }^{10}$, David G. Cook ${ }^{11,12}$, Susan J. Tappan ${ }^{10}$, Sam Gandy 2,3,4,5,13,14, Patrick R. Hof 3,6,13,15, Stephen T. Ahlers ${ }^{7}$ and Gregory A. Elder $2,3,5,13,16$

\begin{abstract}
Cerebral vascular injury as a consequence of blast-induced traumatic brain injury is primarily the result of blast waveinduced mechanical disruptions within the neurovascular unit. In rodent models of blast-induced traumatic brain injury, chronic vascular degenerative processes are associated with the development of an age-dependent posttraumatic stress disorder-like phenotype. To investigate the evolution of blast-induced chronic vascular degenerative changes, Long-Evans rats were blast-exposed $(3 \times 74.5 \mathrm{kPa})$ and their brains analyzed at different times post-exposure by X-ray microcomputed tomography, immunohistochemistry and electron microscopy. On microcomputed tomography scans, regional cerebral vascular attenuation or occlusion was observed as early as $48 \mathrm{~h}$ post-blast, and cerebral vascular disorganization was visible at 6 weeks and more accentuated at 13 months post-blast. Progression of the late-onset pathology was characterized by detachment of the endothelial and smooth muscle cellular elements from the neuropil due to degeneration and loss of arteriolar perivascular astrocytes. Development of this pathology was associated with vascular remodeling and neuroinflammation as increased levels of matrix metalloproteinases (MMP-2 and MMP-9), collagen type IV loss, and microglial activation were observed in the affected vasculature. Blast-induced chronic alterations within the neurovascular unit should affect cerebral blood circulation, glymphatic flow and intramural periarterial drainage, all of which may contribute to development of the blast-induced behavioral phenotype. Our results also identify astrocytic degeneration as a potential target for the development of therapies to treat blastinduced brain injury.
\end{abstract}

Keywords: Animal model, Blast, Brain, Chronic, Vascular, Neurovascular unit, Rat, Vascular pathology, Astrocyte, Tight junctions

\footnotetext{
*Correspondence: miguel.gama-sosa@mssm.edu

${ }^{1}$ General Medical Research Service, James J. Peters Department

of Veterans Affairs Medical Center, 130 West Kingsbridge Road, Bronx, NY 10468, USA

Full list of author information is available at the end of the article
}

\section{Introduction}

Traumatic brain injury (TBI) has been linked to mental health disorders and is considered a risk factor for later development of neurodegenerative disorders [31]. Many veterans from the recent conflicts in Iraq and Afghanistan suffer from chronic neurobehavioral syndromes that include post-traumatic stress disorder (PTSD) [31]. original author(s) and the source, provide a link to the Creative Commons licence, and indicate if changes were made. The images or other third party material in this article are included in the article's Creative Commons licence, unless indicated otherwise in a credit line to the material. If material is not included in the article's Creative Commons licence and your intended use is not permitted by statutory regulation or exceeds the permitted use, you will need to obtain permission directly from the copyright holder. To view a copy of this licence, visit http://creativecommons.org/licenses/by/4.0/. The Creative Commons Public Domain Dedication waiver (http://creativeco mmons.org/publicdomain/zero/1.0/) applies to the data made available in this article, unless otherwise stated in a credit line to the data. 
Indeed, a striking feature in veterans from the most recent war theaters has been the overlap between a history of blast-related mild TBI (mTBI) and PTSD [17, 31, $115,134,140,141]$. While these symptoms may improve, they frequently persist and may worsen with declines driven mainly by worsening PTSD and depression [83]. Although the mechanisms underlying how blast affects human neurobiology are incompletely understood, much evidence suggests that the cerebrovasculature may be particularly vulnerable to blast $[112,131,133]$.

Research in rat models of repetitive low level blast exposure has shown that animals exposed to repetitive low-level blast exhibit chronic cognitive impairment and PTSD-related traits that develop over time [30, 33, 100, $101,123]$. These traits include anxiety, enhanced acoustic startle, altered fear learning and impaired cognition in tests such as novel object recognition. Blast-exposed rats thus model many of the features found in human PTSD. A single predator scent challenge delivered 8 months after the last blast exposure induces additional anxietyrelated changes that are still present 45 days later [101] suggesting that besides inducing PTSD-related traits blast exposure sensitizes the brain to react abnormally to subsequent psychological stressors.

The evolution of the resulting blast-induced cognitive and behavioral phenotypes in rats seems to overlap the development of cerebral vascular degenerative processes. [38-40]. Cerebral blood circulation is essential for normal brain metabolic functions, as it provides oxygen, glucose and other essential metabolites as well as removes metabolic wastes [125]. Increased synaptic activity increases blood flow in brain regions where higher oxygen concentrations are required via neurovascular coupling. The neurovascular unit is composed of endothelial cells, associated blood-brain barrier tight junctions, a basal lamina covered with mural cells (pericytes and smooth muscle), neural cells (astrocytes and neurons) and an extracellular matrix (ECM). The neurovascular unit also encompasses the glymphatic and intramural periarterial drainage systems. The glymphatic CSF waste clearance system utilizes perivascular channels, formed by astroglial cells, to promote elimination of soluble proteins and metabolites from the CNS $[14,61,91]$. In this sytem, CSF enters interstitial spaces after aquaporin 4 (AQP4)-dependent transport through the astroglial cytoplasm, drains into perivenous routes in a direction parallel to the blood flow, and then enters the subarachnoid CSF or bloodstream across cerebral vessels [142]. The intramural periarterial drainage system (IPAD) is a major pathway by which solutes drain from the brain to cervical lymph nodes along the walls of cerebral arteries in a direction counter to the blood flow $[1,56,136]$. In the
IPAD system, parenchymal interstitial fluid (ISF) enters the basal membrane of capillaries and is progressively moved onto the basal membrane of the tunica media of intracerebral arterioles and arteries, and onto the walls of leptomeningeal and internal carotid arteries[5, 19, 142].

Direct consequences of blast exposure include acute damage and chronic structural degeneration of the cerebral vasculature. Early blast-induced vascular injury includes apoptosis of vascular structural elements, capillary strictures, vascular occlusion, blood-brain barrier disruption, vascular rupture, breakdown of the choroid plexus, reduced dilator responses to decreased intravascular pressure, reduced cerebral perfusion, and increased cerebral vascular resistance, $[38,39,63,66,75,113,114]$. These acute events are followed by the development of a chronic secondary pathology characterized by perivascular astrocytic degeneration, luminal collapse, disruption of neurovascular interactions, ECM remodelling, "double-barreled" vessels, intraluminal astrocytic processes, vascular smooth muscle degeneration, vascular occlusion by $\mathrm{CD} 34^{+}$progenitor cells, generalized vascular attenuation, aneurysm formation, vascular fragility and stroke [40]. These combined events can lead to disruptions in cerebral blood circulation that seem to be associated with development of PTSD-related symptoms in blastexposed individuals [31, 33, 83, 102, 132]. Similarly, CSF and ISF drainage in the brain through the glymphatic and IPAD systems along the external perivascular spaces and laminae may also be affected.

In the present research, we used a combination of X-ray microcomputed tomography (micro-CT) with computerized morphological analyses to determine the evolution of vascular structural alterations in the brain circulation of blast-exposed rats. Micro-CT scans revealed regional cerebral vascular attenuation as early as $48 \mathrm{~h}$ post-blast and cerebral vascular attenuation and disorganization at 6 weeks and 13 months post-blast. Immunohistochemical and histological analyses confirmed the presence of previously reported vascular degenerative processes but also revealed at 13 months post-blast the emergence of a vascular pathology characterized by the detachment of the vasculature from the brain parenchyma with affected arterioles at the core of an enlarged paravascular space. This chronic vascular pathology was associated with perivascular-astrocytic degeneration, vascular attenuation, vascular-associated inflammation and vascular remodeling. Our results further document the evolution of blast-induced neurovascular uncoupling due to astrocytic and vascular degenerative processes and provide evidence for a novel pathology that may affect blood circulation and perivascular flow of CSF and ISF through the glymphatic and IPAD systems in the brain. 


\section{Materials and methods}

\section{Animals}

Adult male Long-Evans hooded rats (250-350 g, 10 weeks of age, Charles River Laboratories International, Wilmington, MA, USA) were used. All studies involving animals were reviewed and approved by the Institutional Animal Care and Use Committees of the Walter Reed Army Institute of Research (WRAIR)/ Naval Medical Research Center and the James J. Peters VA Medical Center. Studies were conducted in compliance with the Public Health Service policy on the humane care and use of laboratory animals, the NIH Guide for the Care and Use of Laboratory Animals, and all applicable Federal regulations governing the protection of animals in research.

\section{Blast overpressure exposure}

The Walter Reed Army Institute of Research (WRAIR) shock tube at the Naval Medical Research Center (NMRC, Silver Springs, MD, USA) was used to expose rats to overpressure injury. This apparatus, which simulates the effects of air blast exposure under experimental conditions, has been used in our prior studies to deliver blast overpressure injury to rats [4, 20-22, $25,30,39,40]$. As in previous studies, anesthetized rats were randomly assigned to sham or blast conditions with the head facing the blast exposure without any body shielding, resulting in a full body exposure to the blast wave. The physical characteristics of the blast wave and further details of the blast exposure have been described in detail [4]. Blast-exposed animals received a total of three $74.5-\mathrm{kPa}(10.8 \mathrm{psi})$ exposures, with one exposure administered daily for 3 consecutive days. Control animals were anesthetized and placed in the blast tube but not subjected to a blast exposure. Within 10 days after the last blast exposure, the animals were transferred to the James J. Peters VA Medical Center (Bronx, NY, USA) where all other procedures were performed. Control and experimental cohorts were euthanized at $48 \mathrm{~h}$ ( $\mathrm{n}=6$ per group), 6 weeks ( $n=6$ per group) and 13 months $(n=4$ blast-exposed rats and $n=5$ control rats) post-blast for observation of acute, subacute and chronic effects, respectively.

\section{Behavioral testing}

Some animals used in this study were previously characterized behaviorally [99]. Data from selected animals were used here. Elevated zero maze (EZM) as well as contextual and cued fear conditioning (FC) tests were performed at 40 and 42 weeks post-exposure, respectively [99].

\section{X-ray high-resolution micro-CT scanning}

Rats were anesthetized with $150 \mathrm{mg} / \mathrm{kg}$ ketamine and $30 \mathrm{mg} / \mathrm{kg}$ xylazine and transcardially perfused first with $60 \mathrm{ml}$ of $10 \mu \mathrm{g} / \mathrm{ml}$ heparin in phosphate-buffered saline (PBS), $\mathrm{pH} 7.2$, followed by $250 \mathrm{ml}$ of a $30 \%$ solution of the Brite Vu Special Projects contrast agent supplemented with its enhancer (Scarlet Imaging, Murray UT, USA) maintained at $65{ }^{\circ} \mathrm{C}$. The perfused animals were chilled by immersion in an ice-water bath for $2 \mathrm{~h}$ to gel the intravascular contrast agent. Brains were then dissected, post-fixed overnight in $4 \%$ paraformaldehyde in PBS and maintained in sterile PBS at $4{ }^{\circ} \mathrm{C}$. Brains were scanned at a $7.5-\mu \mathrm{m}$ voxel size using $60 \mathrm{kV}, 166 \mu \mathrm{A} X$-ray settings and a $0.25-\mathrm{mm}$ aluminum filter with exposure time of $508 \mathrm{~ms}$ per frame, with 3 frames averaged at each projection angle with a Bruker SkyScan 1272 micro-CT (Micro Photonics, Allentown, PA, USA). Three-dimensional reconstruction and morphological profiling of the cerebral vasculature was performed with the Bruker's NRecon software or the Vesselucida 360 software (v2018.1.1, MBF Bioscience LLC, Williston, VT, USA) using data obtained from the micro-CT scans and reconstructing the respective three-dimensional vascular networks. For quantitative analyses, automatic reconstruction of the vasculature with the Vesselucida 360 software was performed using identical settings for all animals. A voxel scooping algorithm was applied with the following settings: trace and seed sensitivity set to 80 , medium seed density with refine filter set to 2, maximum gap tolerance. No manual editing was performed. The parameters determined were total vascular length, volume, surface area, length density, the number of isolated vessels, the longest vessel, the number of branching nodes, number of ending vessels and the estimated total tissue volume.

\section{Histological and immunohistochemical analysis}

Coronal sections (50 $\mu \mathrm{m}$ thickness) of the micro-CT scanned brains were prepared with a VT1000S Vibratome (Leica Biosystems, Buffalo Grove, IL, USA). General histology was assessed by hematoxylin-eosin staining of sections. For immunohistochemistry, sections were blocked with $10 \%$ normal goat serum in $50 \mathrm{mM}$ Tris $\mathrm{HCl}$, $\mathrm{pH} 7.6,0.15 \mathrm{M} \mathrm{NaCl}, 0.3 \%$ Triton-X-100 and incubated overnight with the primary antibodies diluted in blocking solution at room temperature. After washing with PBS (6 times for $10 \mathrm{~min}$ each), sections were incubated with the appropriate Alexa (488, 568 and 647)-conjugated secondary antibodies (1:300, ThermoFisher, Waltham, MA, USA) in blocking solution for $2 \mathrm{~h}$. After washing with PBS (6 times for $10 \mathrm{~min}$ each), the sections were mounted with Fluorogel mounting medium (Electron Microscopy Sciences, Hatfield, PA, USA). To visualize 
nuclei, sections were incubated in PBS containing $0.1 \mu \mathrm{g} /$ $\mathrm{ml}$ DAPI (4,6-diamidine-2'-phenylindole dihydrochloride) in the next to last PBS wash. The primary antibodies were a rat monoclonal anti-glial fibrillary acidic protein (GFAP; 1:500, clone 2.2B10, gift of Dr. Virginia Lee, University of Pennsylvania, Philadelphia PA, USA), rabbit anti-GFAP (1:500, G9269, RRID: AB_477035, SigmaAldrich, St Louis, MO, USA), mouse anti- $\alpha$-smooth muscle actin ( $\alpha$ SMA, 1:300, A2547, RRID: AB_262054, Sigma-Aldrich), rabbit monoclonal anti-MMP-9 (1:300, 13667S, RRID: AB_2798289, Cell Signaling Technology, Danvers, MA, USA), mouse monoclonal anti-MMP-9 (1:200, NBP2-80855, RRID: AB_2811297, Novus, Littleton, CO, USA) and rabbit anti-ionized calcium-binding adaptor molecule 1 (Iba1; 019-19741, RRID: AB_839504, Fujifilm Wako Pure Chemical, Osaka, Japan). Vascular staining was performed with anti-collagen type IV antibodies (rabbit anti-rat collagen type IV, 1:300, ab6586, RRID: AB_305584, Abcam, Cambridge, MA, USA) either in the absence or presence of a pepsin treatment $[37,42]$. For the pepsin treatment, sections were incubated with $1 \mathrm{mg} / \mathrm{ml}$ pepsin (Agilent Technologies, Santa Clara, CA, USA) in $3 \%$ acetic acid for $50 \mathrm{~min}$ at $37^{\circ} \mathrm{C}$. Sections were washed once in $0.1 \mathrm{M}$ sodium borate, $\mathrm{pH} 8.5$, washed 5 times with PBS, blocked and stained with antibodies against collagen IV and GFAP in combination with the respective Alexa-conjugated secondary antibodies as described above. For TUNEL staining (terminal deoxynucleotidyltransferase-mediated dUTP nick-end labeling), sections were washed in TBS, permeabilized with $0.1 \%$ Triton X-100 in TBS for $1 \mathrm{~h}$ and washed extensively with TBS. End labeling of DNA with fluorescein-dUTP was performed using a commercial kit (Roche, Indianapolis, IN, USA). After several washes with PBS, the sections were blocked and stained with antibodies against GFAP and ionized calcium-binding adaptor molecule (Iba1) as described above.

\section{Electron microscopy}

Electron microscopy was performed using protocols optimized to study the ultrastructure of the vasculature. Anesthetized rats were transcardially perfused with ice-cold $2.0 \%$ glutaraldehyde, $2 \%$ paraformaldenyde, $0.1 \mathrm{M}$ sodium phosphate ( $\mathrm{pH}$ 7.3). Brains were removed, postfixed in the same fixative as above and stored at $4{ }^{\circ} \mathrm{C}$ until ready for processing. Fixed brains were placed on a rat brain slicer matrix, and coronal slices containing the frontal cortex were excised. Sections were washed in $0.1 \mathrm{M}$ sodium cacodylate buffer, pH 7.2 and postfixed with $1 \%$ osmium tetroxide in $0.1 \mathrm{M}$ sodium cacodylate buffer, $\mathrm{pH}$ 7.2. Sections were washed again in cacodylate buffer, dehydrated through graduated ethanol (70-100\%) and propylene oxide series, and resin-infiltrated with Epon (Electron Microscopy Sciences, Hatfield, PA, USA). Material was polymerized in a vacuum oven at $60{ }^{\circ} \mathrm{C}$ for $48 \mathrm{~h}$. Semi-thin $(1 \mu \mathrm{m})$ Toluidine blue-stained sections were used to identify the regions of interest. Ultrathin sections $(80 \mathrm{~nm})$ were cut with a diamond knife on a Leica UCT ultramicrotome and mounted on copper grids using a Coat-Quick adhesive pen (Electron Microscopy Sciences). Sections were counterstained with uranyl acetate and lead citrate. Frontal cortical sections were imaged on a Hitachi 7700 electron microscope (Hitachi, LTD., Tokyo, Japan) and photographed with an Advantage CCD camera (Advanced Microscopy Techniques, Danvers, MA, USA). Image brightness and contrast were adjusted using Adobe Photoshop 2021 software (version 22.3.1; Adobe, Inc., San Jose, CA, USA).

\section{Zymography of vascular metalloproteases}

Enriched cerebral vascular fractions were prepared as previously described [40]. Blast-exposed and control rats (6 weeks post-blast) were euthanized by $\mathrm{CO}_{2}$ inhalation. Brains were cleaned of meninges and homogenized in cold 18\% dextran in PBS (10 ml/g of tissue) using a Potter-Elvejehm homogenizer with a loose-fit Teflon pestle (6-8 strokes at low speed, setting 2 of a Wheaton overhead stirrer, Millville, NJ, USA). Each homogenate was overlayed over an equal volume of Ficoll-Paque PLUS ${ }^{\text {TM }}$ (GE Healthcare Life Sciences, Marlborough, MA, USA) and centrifuged for $30 \mathrm{~min}$ at $1500 \times \mathrm{g}$ and $4{ }^{\circ} \mathrm{C}$. Vascularenriched pellets were resuspended in PBS, washed twice with PBS, and stored at $-80{ }^{\circ} \mathrm{C}$ [40]. Enriched vascular fractions were lysed in $10 \mathrm{mM} \mathrm{NaPO}_{4}, \mathrm{pH} 7.4,150 \mathrm{mM}$ $\mathrm{NaCl}, 2 \mathrm{mM}$ EDTA, $1 \%$ Triton X-100, 0.5\% sodium deoxycholate, and $1 \%$ sodium dodecyl sulfate (SDS) supplemented with protease and phosphatase inhibitor cocktails 2 and 3 (Sigma-Aldrich, St Louis, MO, USA). Lysates were centrifuged at $15,000 \times \mathrm{g}$ for $15 \mathrm{~min}$, and the protein concentration in the supernatants was determined with the BCA reagent (ThermoFisher, Waltham MA, USA). Heat-denatured samples containing $20 \mu \mathrm{g}$ of protein were electrophoresed through a $10 \%$ polyacrylamide gel containing $0.1 \%$ gelatin (Novex 10\% Zymogram Plus Gelatin Protein Gel, Thermo Fisher Scientific, Waltham, MA). After protein renaturation, incubation, and staining with Brilliant Blue, gelatinase activity was visualized as cleared bands reflective of gelatin hydrolysis.

\section{Statistical analyses}

Statistical differences were evaluated with unpaired $t$-tests using Prism 7.0 software (GraphPad, La Jolla, CA). Statistical significance was set at an $\alpha$ level of 0.05 . 


\section{Results}

\section{Cerebral vascular disorganization revealed by micro-CT scanning}

The evolution of the blast-induced chronic cerebral vascular pathology was investigated by X-ray high-resolution micro-CT scanning of brains of blast-exposed rats at 48 h, 6 weeks and 13 months post-blast exposure following perfusion with the Brite $\mathrm{Vu}$ contrast agent. Figure 1 shows representative maximum intensity projection (MIP) images of the volume-rendered brain vasculature of control and blast-exposed animals at the three time points. In Fig. 2, magnified high resolution micro-CT images are shown which reveal that in addition to large and medium sized arteries, smaller vessels including precapillary arterioles and possibly venules could be imaged. Quantitative analyses of the total cerebral vasculature (Fig. 3) did not show significant differences in major parameters, including length, surface area and volume, of the cerebral vasculature between blast-exposed and controls at any of the time points.

Nevertheless, post-blast imaging analyses of regional optical coronal sections (3.75-mm thickness) revealed stochastic vascular alterations in blast-exposed animals with attenuation, occlusion or disruption of the regular radial patterns visible in the brains of control animals. For example, Fig. 4a shows a rat brain from the $48 \mathrm{~h}$ post-blast cohort with vascular alterations in the hippocampal and posterior cortical regions of the left cerebral hemisphere, most likely the result of occlusion or attenuation of the posterior branches of the MCA as well as collateral branches of the posterior cerebral artery (PCA) and the anterior choroidal artery (AChA).

At 6 weeks and 13 months post-blast, major alterations and disorganization of the cerebral vasculature were also observed. At 6 weeks post-blast, half of the blast-exposed rats $(3 / 6)$ exhibited vascular alterations. Figure $4 \mathrm{~b}$ shows attenuation of the vasculature irrigating the cingulate, motor, somatosensory, and retrosplenial cortex most likely involving the rostral branches of the MCA. At 13 months post-blast, cerebral vascular alterations were clearly observed in all blast-exposed animals (4/4) and none of the sham control rats (5/5) (Fig. 4c). In these animals, tissue tears, induced by the initial blast wave injury were clearly visible [39] (Fig. 4c). For example, in Fig. 4c lesions extend through the perirhinal or piriform cortical regions as well as the external capsule and amygdala and are similar to those described in previous blast-exposed cohorts $[38,39]$. In the animal shown in Fig. 4c, blastinduced injuries resulted in bilateral amygdalar lesions. Interestingly, this animal which had been previously characterized behaviorally [95] was a relative outlier among blast-exposed animals in an elevated zero maze

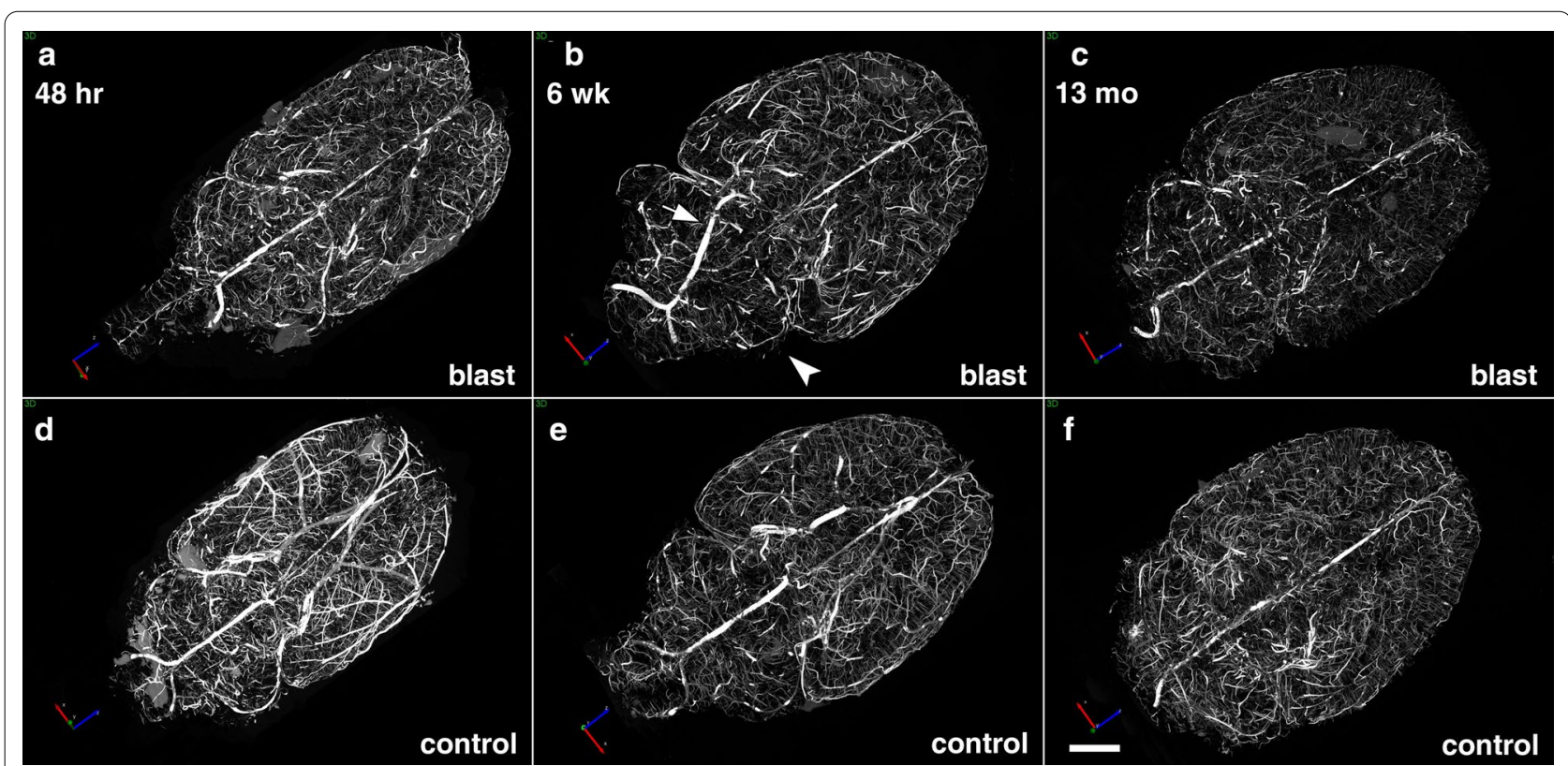

Fig. 1 Micro-CT scanning of brains of blast-exposed and control rats. Blast-exposed and control rats were transcardially perfused with the Brite Vu contrast agent at $48 \mathrm{~h}, 6$ weeks and 13 months after blast exposure. Brains were scanned at a resolution of $7.5 \mu \mathrm{m}$ using $0.3^{\circ}$ rotational steps of view around $360^{\circ}$ and three-dimensional reconstructions were prepared with Bruker's NRecon software before visualization with Bruker's CTVox 3D visualization software. Representative maximum intensity projection (MIP) images show dorsal view of volume-rendered brain vasculature from blast-exposed (a-c) and control (d-f) rats. Arrow in $\mathbf{b}$ shows an apparently displaced basilar artery in this blast-exposed animal. Arrowhead in $\mathbf{b}$ indicates a focally hypoperfused region within the cerebellum. Scale bar, $2 \mathrm{~mm}$ 


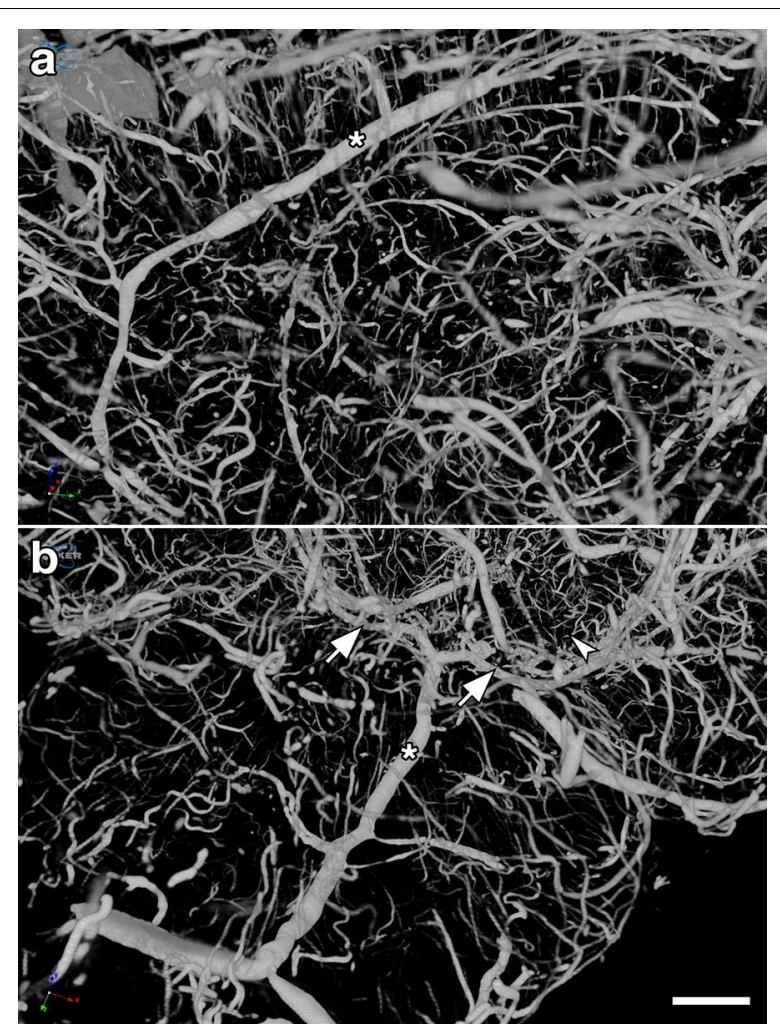

Fig. 2 High resolution images of micro-CT scanning of the rat cerebral vasculature. a lateral view of the left middle cerebral artery $\left(\mathrm{MCA},{ }^{*}\right)$ and $(\mathbf{b})$, posterior view of the basilar $\left(^{*}\right)$ and the posterior cerebral arteries (arrows) from a control rat, 13 months post-sham exposure. Note visualization of large, medium and small vessels including what are likely precapillary arterioles (arrowhead). Scale bar, $1 \mathrm{~mm}$

(EZM; Fig. 5a-c) making more open arm entries (Fig. 5a) as well as spending more time in the open arms (Fig. 5b) and exhibing a short cross arm latency (Fig. 5c). In a fear conditioning task (Fig. 5d-f), this animal showed no freezing responses during the training session (Fig. $5 \mathrm{~d}$ ) but exhibited relatively normal freezing during the contextual (Fig. 5e) and cued fear testing (Fig. 5f). In Fig. 5, behavioral data from the control animal illustrated in Fig. 4f is shown for comparison.

\section{Perivascular astrocytic degeneration, loss, and mislocalization}

We previously described the blast-induced degeneration of astrocytes surrounding the cerebral vasculature as already ongoing at 6 weeks post-exposure [40]. To evaluate the astroglial coverage of the brain vasculature at 13 months post-blast, sections of control and blastexposed animals were analyzed first by electron microscopy (Fig. 6). Electron micrographs showed long-term blast-induced effects on perivascular astrocytes including swelling and degeneration of astrocytic endfeet in the brain cortical vasculature. The processes of astrocytic endfeet in an advanced degenerating phase were devoid of cellular organelles (Fig. 6). Some small vessels and arterioles had enlarged paravascular spaces that reflected the loss of perivascular astrocytes bridging the parenchymal neuropil and the vessel. Apoptotic perivascular astrocytes and microglia were detected by Tunel staining associated with vasculature with enlarged paravascular spaces (Fig. 7). These observations are in agreement with the development of an unusual vascular pathology observed in two additional animals from the 13 months post-blast cohort (Fig. 8). This pathology, characterized mainly by the presence of vascular segments within enlarged paravascular spaces (Fig. 8), often affected mainly one cerebral hemisphere. The brain regions affected generally included the cerebellum, hypothalamus, thalamus, neocortex, hippocampus, basal ganglia, piriform cortex and amygdala (Fig. 8a). Vascular reconstructions of micro CT optical sections showed that brain regions with enlarged paravascular spaces also showed drastic reductions of micro-CT-traced vessels. As an example, Fig. $8 \mathrm{a}, \mathrm{b}$ shows that enlarged paravascular spaces in the hypothalamus, ventral thalamus and, most strikingly, the amygdala and piriform cortex have a clear reduction of micro-CT-traced vessels. The lack of vascular tracing within regions with enlarged paravascular spaces is indicative of vascular hypoperfusion or attenuation and could be a consequence of lack of vascular tone regulation due to perivascular astrocytic degeneration. In behavioral testing this animal exhibited an anxiety phenotype in an EZM that was similar to other blast- exposed animals. (Fig. 5a-c). In fear conditioning, it exhibited a training curve (Fig. 5d) relatively similar to other blast-exposed animals but showed increased freezing in contextual (Fig. 5e) and cued (Fig. 5f) fear testing. Histochemical, immunohistochemical and electron microscopic analyses showed that the vasculature enclosed within the enlarged paravascular spaces involved mainly arterial vessels (Fig. $8 \mathrm{c}-\mathrm{f}$ ) and in some of these vessels the adventitial layer could be found detached (Fig. 8d, f).

As previously reported [3, 38, 40], some of the arterioles presented increased tortuosity and structural smooth muscle alterations as strictures and tortuosity could result in loss of smooth muscle (Fig. 9e). GFAP and collagen type IV immunostaining revealed collagen IV degradation associated with loss of perivascular astrocytes in the affected vasculature (Figs. 7, 9, 10). Compared to unaffected brain regions, loss of vascular collagen IV immunostaining was seen in areas with enlarged paravascular spaces (Fig. 9a-f). Although perivascular astrocytes were lost in the affected vasculature and immediate surrounding parenchyma, remains of 


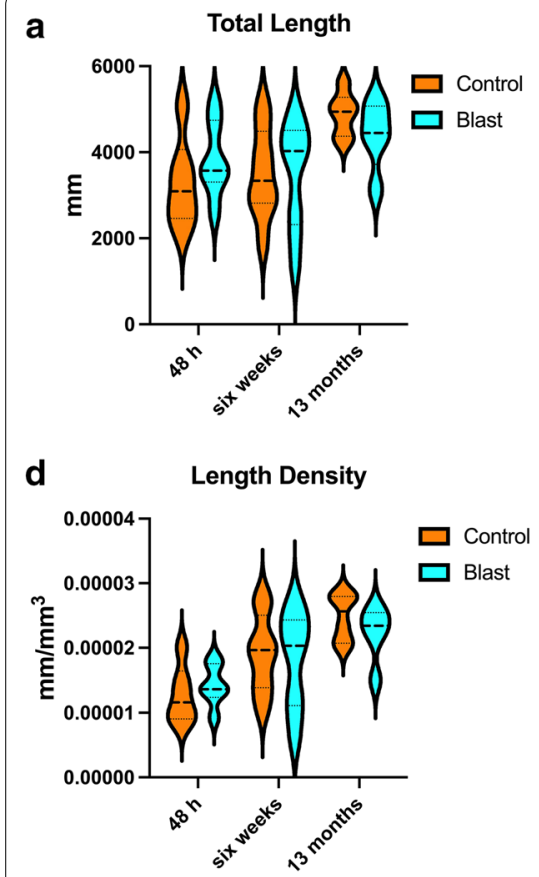

g

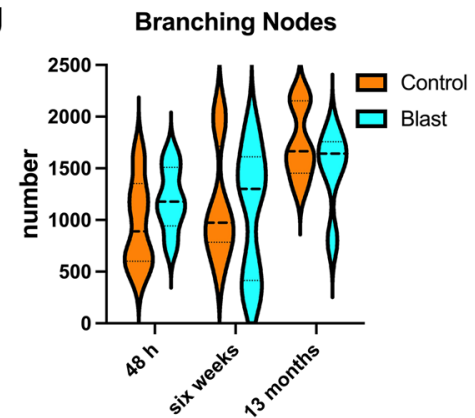

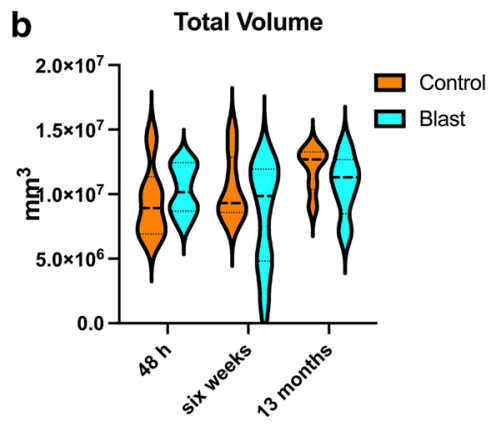

e

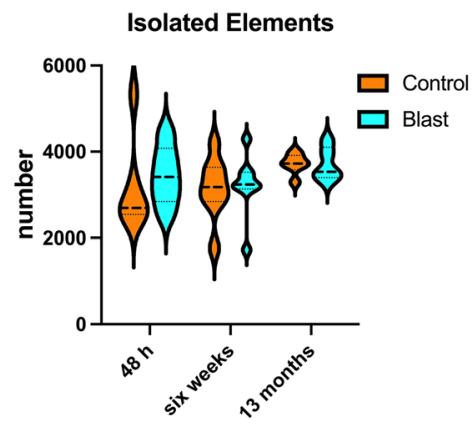

h

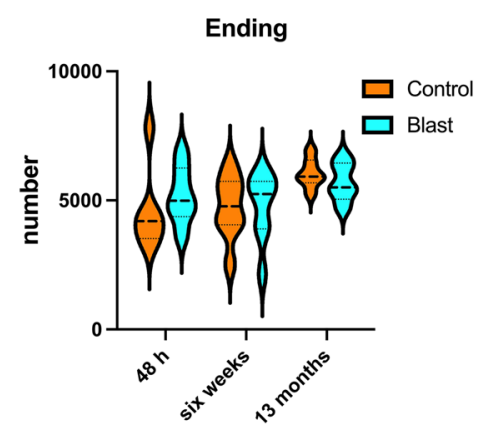

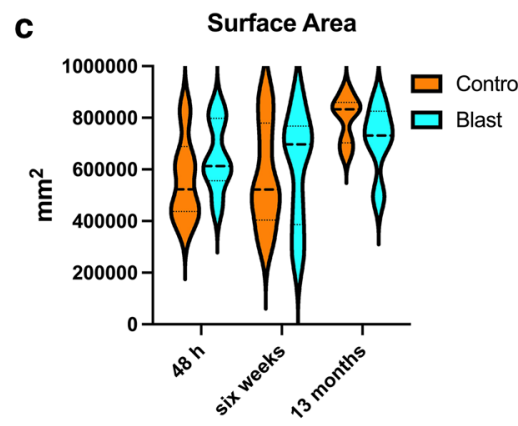

f

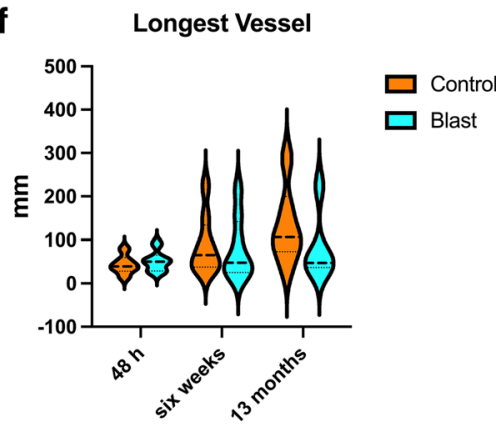

i

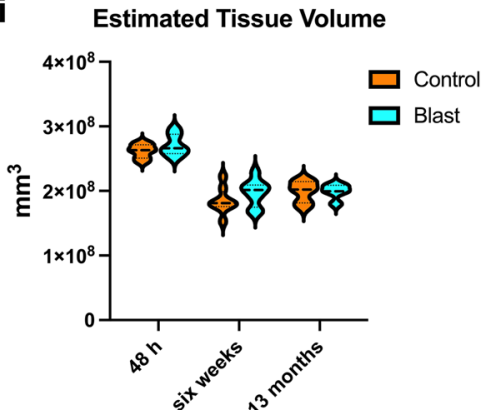

Fig. 3 Quantification of X-ray high-resolution micro-CT scanning. Parameters were determined using the software Vesselucida Explorer (v2018.1.1, MBF Bioscience LLC, Williston, VT, USA). Shown is total vascular length $\mathbf{a}$, volume $\mathbf{b}$, surface area $\mathbf{c}$, length density $\mathbf{d}$, the number of isolated vessels $\mathbf{e}$, the longest vessel $\mathbf{f}$, the number of branching nodes $\mathbf{g}$, ending vessels $\mathbf{h}$ and the estimated total tissue volume $\mathbf{i}$. Data is shown as violin plots. There were no statistically significant differences between the blast-exposed and sham-exposed (control) cohorts at any of the times studied post-exposure

$\mathrm{GFAP}^{+}$astrocytic feet could be seen remaining attached in some vessels (Fig. 9c).

\section{Remodeling of the adventitial ECM}

In addition to the loss of perivascular astrocytes, some vessels showed mislocalization of astrocytes. We previously reported the presence of intravascular $\mathrm{GFAP}^{+}$ astrocytic processes in the cerebral vasculature of animals at 6 weeks post-blast exposure [40]. At 13 months post-blast, confocal microscopy identified astrocytes under the adventitial ECM scaffold (Fig. 10f) and in the lumen (Fig. 11c, d). This implies ECM remodeling by adventitial fibroblasts along with perivascular astrocyte repositioning within the vascular internal structural layers. The presence of intraluminal astrocytes or astrocytic processes may identify non-functional degenerating vasculature into which perivascular astrocytes and/or their processes could extend.

Adventitial fibroblasts become involved in vascular remodeling via their regulation of ECM degradation mediated by MMPs and tissue inhibitors of metalloproteinases (TIMPs). The presence of vascular protease activity in rats at 10 months post-blast has previously been demonstrated by the immunostaining of the cerebral vasculature with collagen type IV antibodies without prior protease treatment [38]. Similarly, at 13 months post-blast, the cerebral vasculature was extensively stained with anti-collagen type IV 


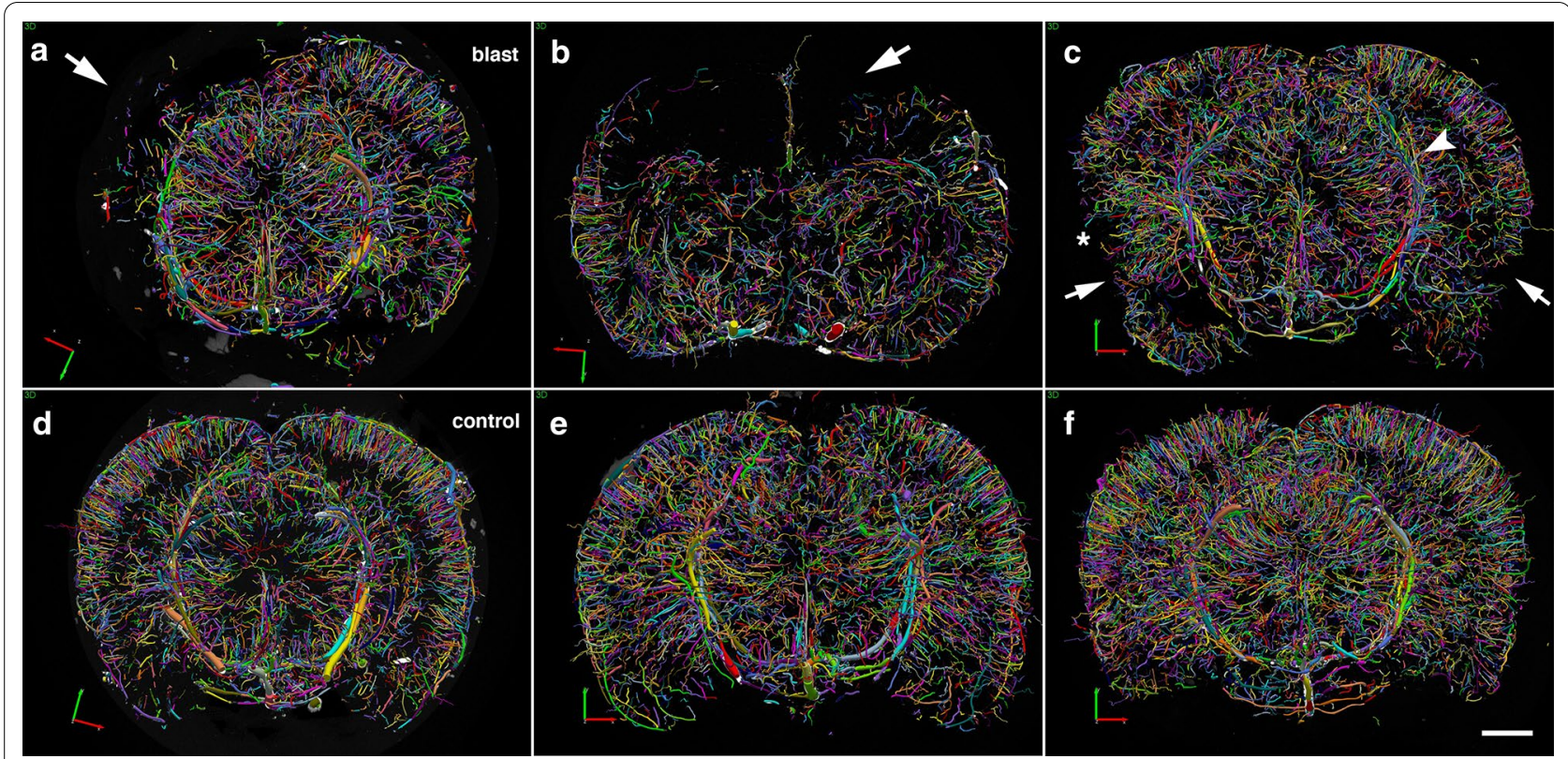

Fig. 4 Acute, subacute and chronic vascular hypoperfusion and disorganization in the brains of blast-exposed rats. Brain micro-CT coronal optical sections (3.75 mm thickness) of blast-exposed and control rats perfused with the Brite-Vu-contrasting agent and reconstructed with the Vesselucida 360 software. a 48 h post-blast; $\mathbf{b} 6$ weeks post-blast; $\mathbf{c} 13$ months post-blast; $\mathbf{d}-\mathbf{f} 48 \mathrm{~h}-, 6$ weeks- and 13 months sham controls, respectively. Optical sections $\mathbf{a}$ and $\mathbf{d}$ correspond approximately to coordinates interaural $4.06-7.56 \mathrm{~mm} ; \mathbf{b}$ and $\mathbf{e}$ to interaural 6.36-9.86 mm; $\mathbf{c}$ and $\mathbf{f}$ to interaural $4.06-$ $7.56 \mathrm{~mm}$ [97]. Note hypoperfused areas in the brains of blast-exposed rats relative to controls. The arrow in $\mathbf{a}$ indicates hypoperfusion of the entire left hemispheric cortical and hippocampal areas. Affected areas in $\mathbf{b}$ include the somatosensory, visual, motor and retrosplenial cortical regions whose circulation is derived from branches of the middle cerebral artery (MCA). In c blast-induced cerebral disorganization includes focal tears across the amygdala and adjacent cortical regions (arrows), attenuation of arteries involved in posterior circulation (arrowhead) and hypoperfusion of cortical areas (*). Scale bar, $1.5 \mathrm{~mm}$

antibodies in the absence of a protease treatment. In age-matched controls, collagen type IV vascular staining was very limited (Fig. 12a, b).

Increased levels of brain MMP-9, MMP-9 mRNA, and plasma MMP-9 have been observed acutely after a $130-\mathrm{kPa}$ blast exposure in association with bloodbrain barrier dysfunction [66]. Gelatin zymography of purified cerebral vascular fractions showed that levels of MMP-2 and MMP-9 could also be increased at 6 weeks post-blast exposure (Fig. 13a). At 13 months post-blast, the cerebral vasculature showed a reduction in collagen type IV immunoreactivity in some regions (Fig. 9), smooth muscle expression of MMP-9 (Fig. 13b-e) and thickening of the vascular laminae (Fig. 14). In addition, vascular IgG leakage was detected at this age suggesting that the peripheral circulation might be an additional source of proteases affecting the vasculature (Fig. 13f, g). These results illustrate the developing chronic vascular remodeling of the ECM in the vascular scaffold after blast exposure.

\section{Vascular-associated neuroinflammation}

Microglial activation is a brain pathology hallmark that occurs in response to injury through damage-associated molecular patterns and removal of immunosuppressive signals. Electron microscopic observations at 13 months post-blast identified periarteriolar microglia with intracellular cholesterol crystals next to degenerating astrocytic endfeet (Fig. 15a, b, d).

At 13 months post-blast, activated microglia were predominantly Iba $1^{+}$types 3 and 4 microglia (relative large soma and short ramifications) in areas neighboring affected vessels (Figs. 16, 17). Electron microscopy also showed that these vascular-associated microglia harbored dilated endoplasmic reticulum (Fig. 15e). Activated microglia tended to cluster together and were frequently observed in contact with the affected vasculature. Figure $16 \mathrm{a}-\mathrm{c}$ shows a tortuous tract of the pericallosal artery within the corpus callosum with clustered $\mathrm{Iba}^{+}$activated microglia. Similarly, Fig. 17 also shows activated microglia next to affected vasculature. Evidence for perivascular microglial phagocytosis of astrocytes was obtained in confocal optical sections as intracellular 


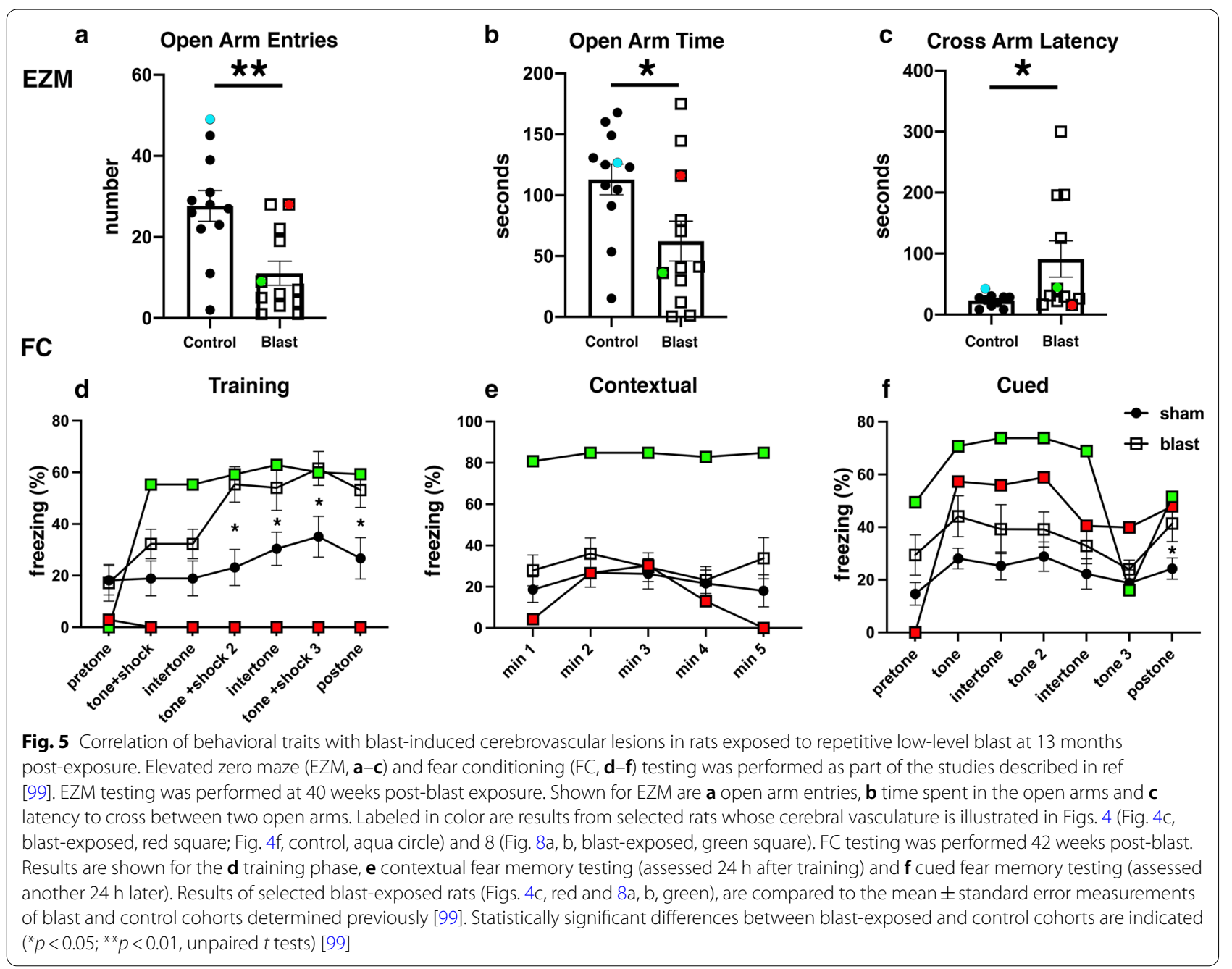

$\mathrm{GFAP}^{+}$material was found within perivascular $\mathrm{Iba}^{+}$ activated microglia (Figs. 16d-f, 17c, d). Small perivascular patches of activated microglia expressing high levels of MHCII (MHCII ${ }^{+}$, characteristic of M1 microglia), were also observed in the brain of a blast-exposed animal. Some perivascular microglia were visibly undergoing apoptosis as seen by their TUNEL staining (Fig. 7) and presence of apoptotic blebs (Fig. 18). Furthermore, Iba $1^{+}$ processes from activated perivascular microglia seemed to transverse the nucleus of neighboring Lochkern-like vascular cells (Fig. 18).

\section{Discussion}

A rat model of low-level blast exposure

We have been studying a rat model developed to mimic blast exposures associated with mild human TBI [4]. Because multiple blast exposures were common among veterans returning from Iraq and Afghanistan [31], we used a design in which rats received three blast exposures, delivered one per day on 3 consecutive days. Under the blast exposure conditions utilized here $(74.5 \mathrm{kPa}, 10.8$ psi), there is no post-exposure apnea, mortality is rare and there are no pulmonary hemorrhages or other lung pathology. During the blast overpressure exposures head motion is restricted to minimize rotation/acceleration injury. Control (sham-exposed) animals are treated identically including receiving anesthesia and being placed in the blast tube, but are not blast-exposed. The lack of histological evidence for coup/contrecoup injuries or generalized brain tissue damage supports the mild nature of the injury and lack of significant rotation/acceleration injury $[4,30]$.

\section{Blast exposure affects the cerebral vasculature}

The high metabolic demand of the brain requires tight coordination between neuronal activity and blood flow $[10,23,29,48,58]$. Because gliovascular and 

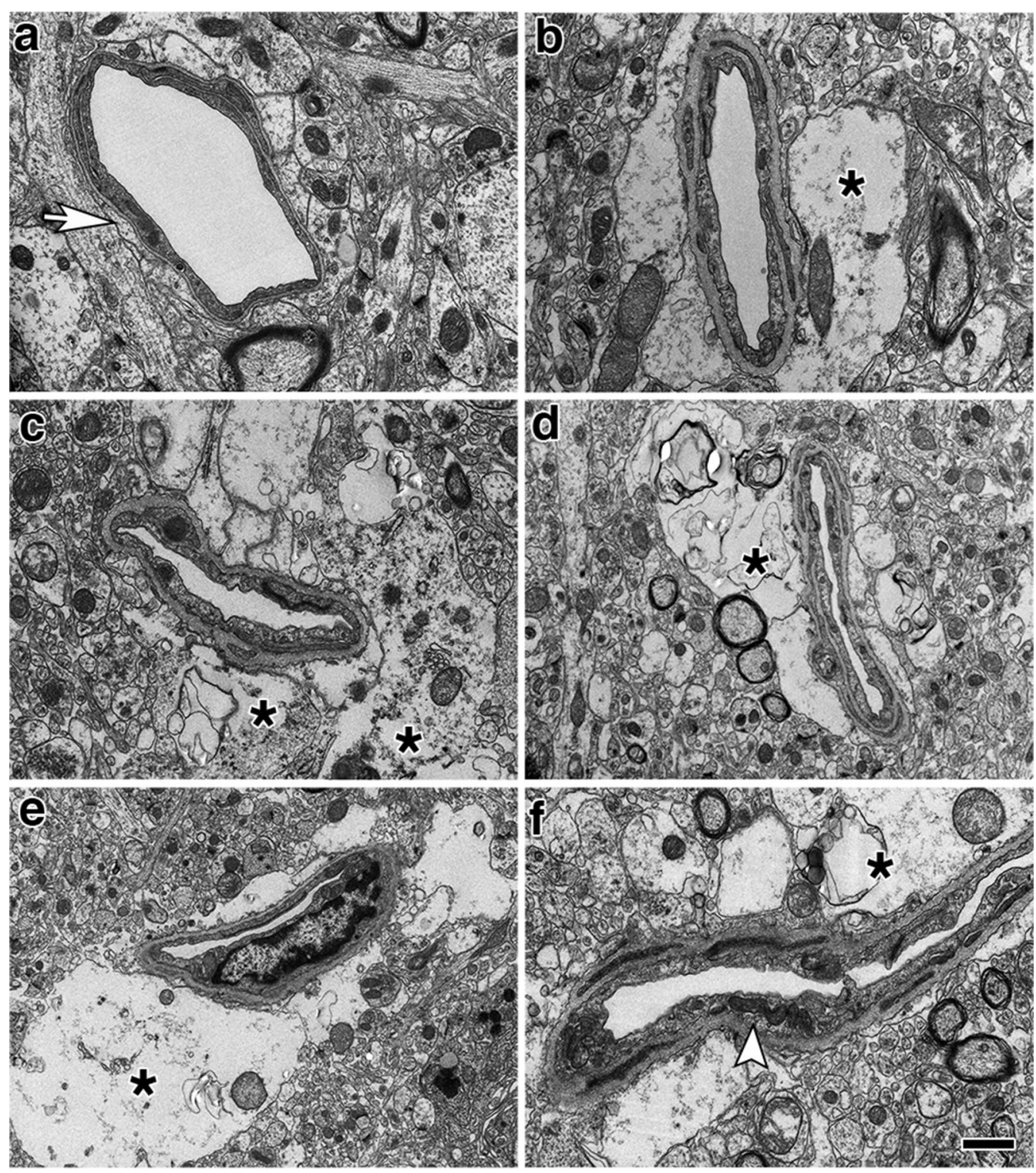

Fig. 6 Astrocytic degeneration in the rat brain 13 months post-blast exposure. Electron micrographs of frontal cortical arterioles from control a and blast-exposed $\mathbf{b}-\mathbf{f}$ rats. $\left(^{*}\right)$ indicates swollen astrocytic feet at different stages of degeneration. Arrow in $\mathbf{a}$ indicates a normal astrocytic endfoot. Arrow head in $\mathbf{f}$ shows an elongated mitochondria in an endothelial cell. Scale bar, $1 \mu \mathrm{m} \mathbf{a}-\mathbf{c}, \mathbf{f} ; 1.5 \mu \mathrm{m} \mathbf{d} ; 2 \mu \mathrm{m} \mathbf{e}$

neurovascular interactions control cerebral blood flow at multiple levels, their disruption following blast exposure would be expected to affect cerebral autoregulation. Morphological, biochemical and functional studies of humans and animal models have identified the vasculature as a primary target for blast-induced tissue damage $[2,6,32,38-41,45,50,54,63,66,75,82,112-114,116$, 130-133, 138]. Considerable evidence supports the concept of a thoracic effect whereby pressure waves transmitted through the systemic circulation damage the brain providing an additional mechanism for why blood vessels and perivascular cellular elements may be particularly susceptible to blast injury $[116,117]$. A summary timeline of cerebral pathological alterations in acute, subacute and chronic cohorts are shown in Fig. 19.

In the present study, we investigated the evolution of blast-induced vascular disease using a rat model. Notably, the general structure of the circulatory system of rats is comparable to that of humans, with the brain deriving its arterial blood supply from the extracranial carotid and vertebral arteries. The internal carotid arteries and their branches supply the anterior two-thirds of the cerebral 


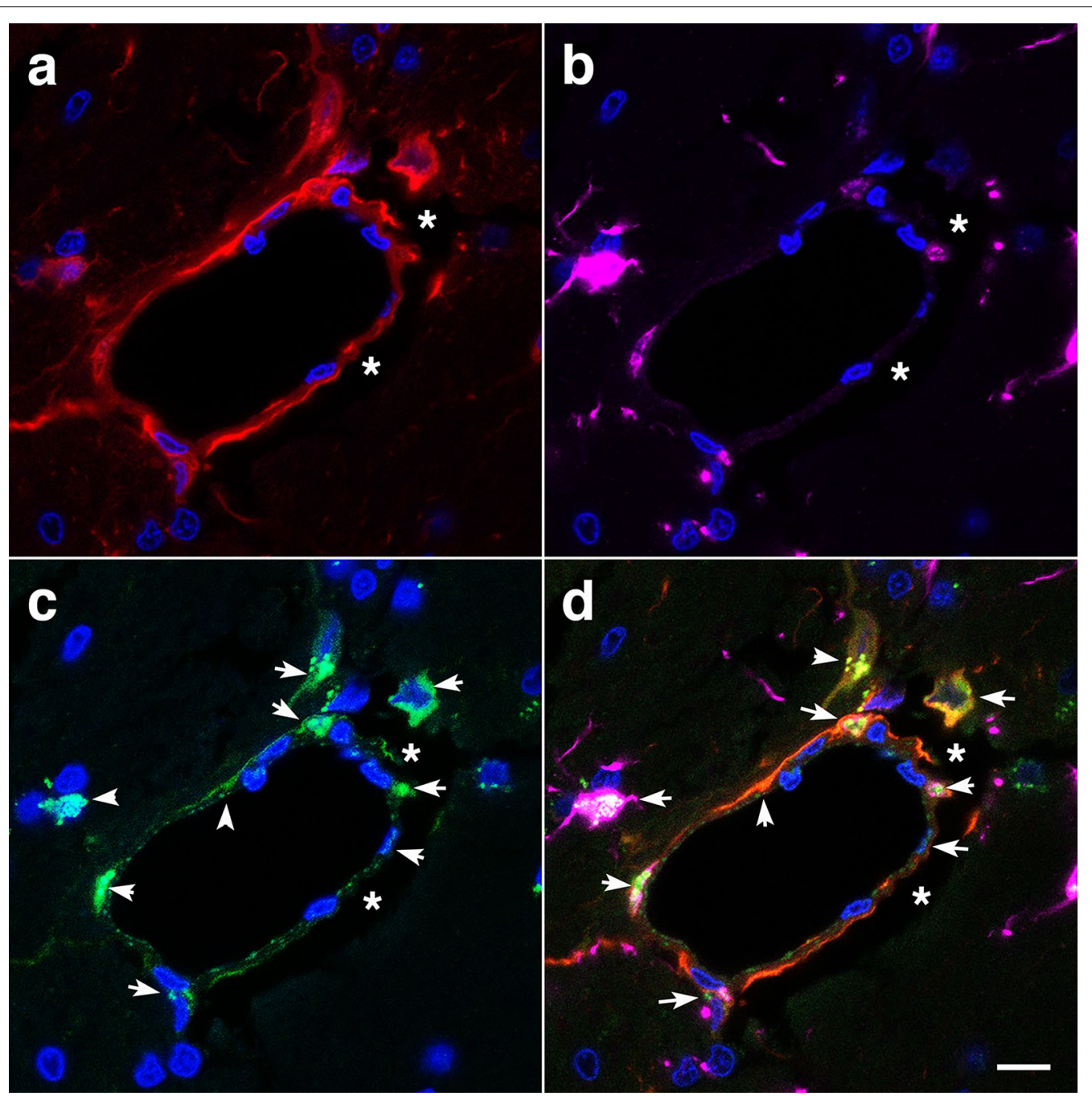

Fig. 7 Perivascular apoptosis in a vessel with enlarged paravascular spaces. A hypothalamic vessel showing perivascular astrocytes (a, GFAP, red) and microglia (b, Iba1, magenta) analyzed by TUNEL staining (c, green), merged $\mathbf{d}$. DAPI (blue). (*) denotes the enlarged paravascular spaces. TUNEL staining of perivascular astrocytes and of astrocyte-associated microglia is indicated by arrows. Scale bar, $25 \mu \mathrm{m}$ a-d

hemispheres, including the deep white matter and basal ganglia. The vertebral arteries that fuse into the basilary artery and its branches supply the remaining posterior and medial regions of the hemispheres, most of the diencephalon, brainstem, cerebellum, and cervical spinal cord. Blast exposure leads to prolonged impairment in vasoreactivity as well as changes in the structure, integrity and phenotype of cerebral blood vessels and astrocytes $[2,40,63,66]$. Localized blast-induced vascular injury may be a determinate of the brain areas where functional alterations will occur.

\section{Blast-induced vascular alterations}

Micro-CT is becoming a widely used method for the accurate resolution of three-dimensional cerebral vascular structures and quantitative volumetric measurements $[28,145]$. By applying this methodology in combination with computerized morphometry, we performed quantitative analyses of the brain vasculature of blastexposed and control rats at different time points postexposure reflecting acute, subacute and chronic periods.

Statistical analyses did not show significant differences in the vascular length, surface area and volume between blast-exposed and control groups at any time point when parameters were analyzed across the whole brain. However, micro-CT analysis allowed the visualization of regional blast-induced alterations of the cerebral vasculature. For example, a rat brain examined $48 \mathrm{~h}$ post-exposure showed vascular attenuation in the left hemisphere, affecting the hippocampus as well as the retrosplenial, 


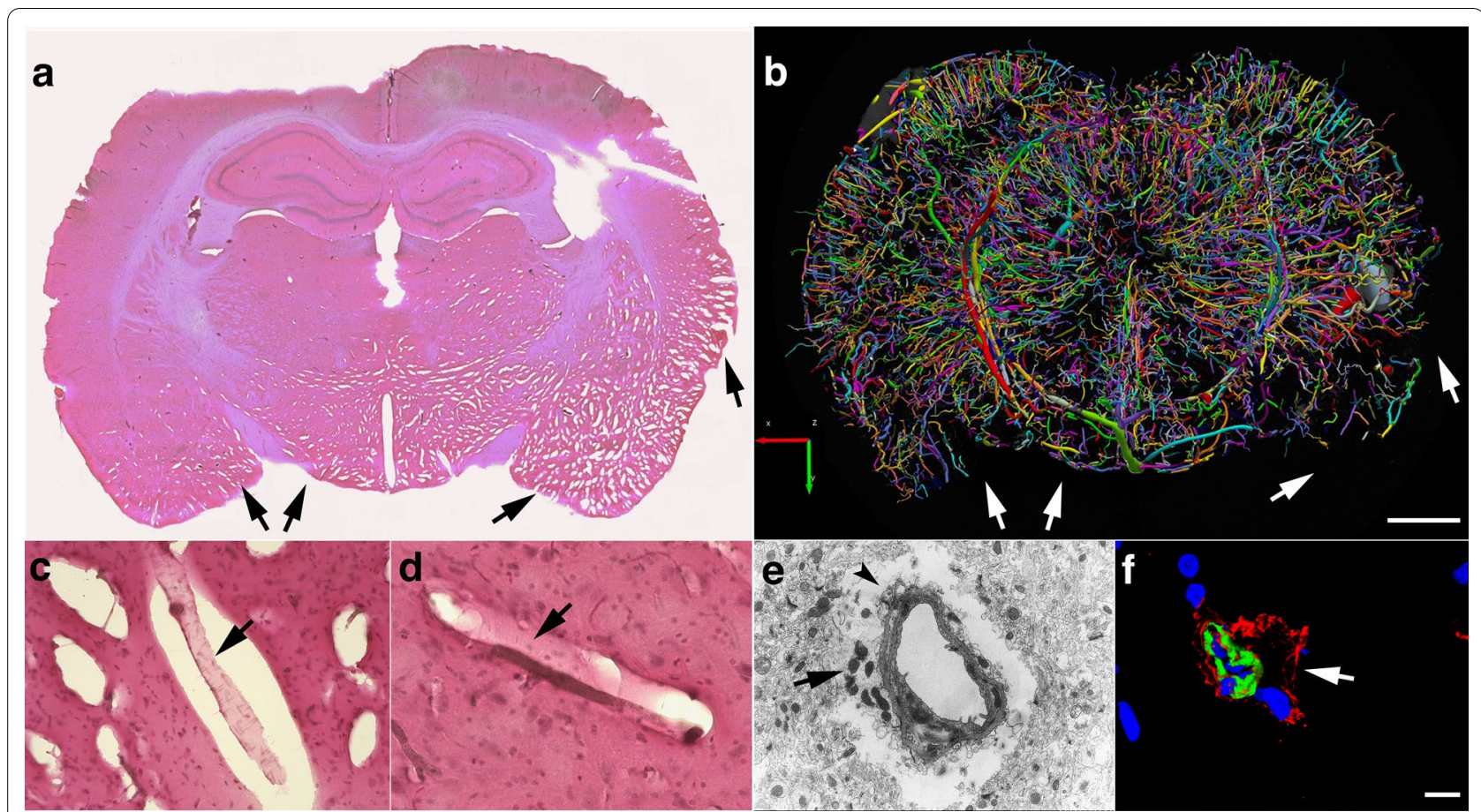

Fig. 8 Chronic enlargement of paravascular spaces in the brain at 13 months post-blast exposure. Coronal sections from a blast-exposed animal were stained with hematoxylin and eosin $\mathbf{a}, \mathbf{c}, \mathbf{d}$. The general pathology showing enlarged paravascular spaces is shown (arrows in $\mathbf{a}$ ). In this animal, the histopathology is mostly concentrated in one hemisphere and involves the amygdala, insula, piriform cortex, ventral thalamus and hypothalamus. In the contralateral hemisphere, pathological changes are limited to the piriform cortex and hypothalamus. Panel $\mathbf{b}$ shows the reconstructed cerebral vasculature from a $3.75 \mathrm{~mm}$ micro-CT optical section that includes the region shown in $\mathbf{a}$. The vascular reconstruction b mirrors the enlarged paravascular spaces seen in panel a where the hypothalamus, ventral thalamus and, most strikingly, the amygdala and piriform cortex show reduced perfused vessels (arrows) and enlarged paravascular spaces. The lack of vascular tracing (arrows in $\mathbf{a}, \mathbf{b}$ ) suggests hypoperfusion of the corresponding areas and could be a consequence of perivascular astrocytic degeneration. Panel c shows a detached artery (arrow) within an enlarged paravascular space in the ventromedial hypothalamus. Panel $\mathbf{d}$ shows a constricted artery (arrow) in the ventral posteromedial nucleus from which the adventia has been detached. Panel e shows an electron micrograph of a cortical arteriole barely attached to the parenchyma through a few degenerating astrocytic endfeet (arrow). Arrowhead in e shows enlarged paravascular space. Panel $\mathbf{f}$ shows immunohistochemical analyses of a hypothalamic arteriole with a disrupted smooth muscle layer (a-SMA ${ }^{+}$, green) and detached adventitia (col IV ${ }^{+}$, red); DAPI, blue. Scale bars, $2 \mathrm{~mm}$ a, b; $40 \mu \mathrm{m} \mathbf{c}$, d; $1.5 \mu \mathrm{m} \mathbf{e} ; 15 \mu \mathrm{m} \mathbf{f}$

visual, auditory, perirhinal and piriform cortical regions as well as part of the amygdala. This involved, most likely alterations in the anterior and posterior circulations, maybe through occlusion or attenuation of the posterior branches of the MCA as well as collateral branches of the PCA and the AChA. At 6 weeks and 13 months post-blast exposure, animals also presented regional alterations and disorganization in the cerebral vasculature. Lesions in the brain (tissue tears) associated with vascular alterations (vascular disorganization and hypoperfusion) correlated with behavioral observations as a rat with bilateral amygdalar lesions, cortical hypoperfusion and hippocampal vascular disorganization failed to express the characteristic chronic anxiety-related behaviors of blast-exposed animals.

We previously described blast-induced vascular attenuation/occlusion due to amorphous cellular debris, vascular collapse due to the negative pressure generated by the fast movement of blast-displaced blood, vascular "pinching" where opposite sides of the vascular walls adhere to each other, occlusion by $\mathrm{CD} 34^{+}$cells, tortuosity and structural alterations in the arterial muscle layer $[3,38$, 40].

\section{Dissociation of the vasculature from the neuropil and vascular remodeling}

Electron microscopy and morphological analyses of brain samples from the 13 months post-exposure cohort showed development of a unique pathology characterized by dissociation of arterioles from the brain parenchyma (neuropil) and perivascular astrocytic degeneration. The arterial/arteriolar wall is a three-layered structure composed of an intima (endothelial cells), media (smooth muscle cells) and adventitia (fibroblasts, nerves, progenitor and immune 


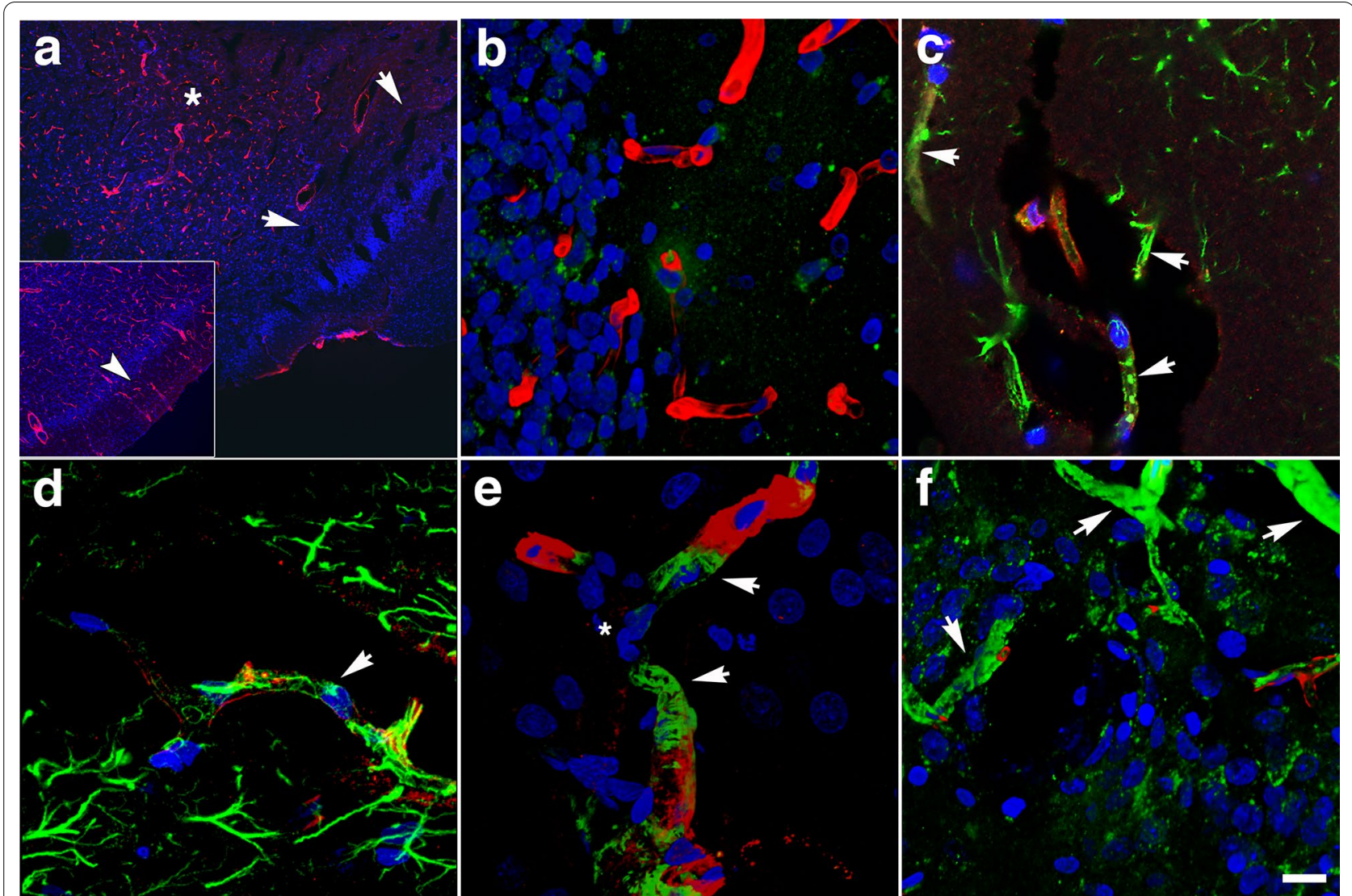

Fig. 9 Loss of vascular collagen type IV in brain regions with enlarged paravascular spaces in rats at 13 months post-blast exposure. Coronal brain sections of a blast-exposed rat were treated with pepsin to unmask the collagen type IV epitopes and stained with rabbit polyclonal antibodies against collagen type IV (red). a Lack of collagen type IV immunostaining (red) in the piriform cortical region exhibiting large paravascular spaces (arrows). Note the normal collagen IV staining (red) in the adjacent areas (asterisk). The inset in a corresponds to a section from a control animal showing normal collagen type IV staining (arrow) of the vasculature in the piriform cortex and neighboring regions. $\mathbf{b}$ Normal vascular collagen type IV staining of the hippocampal vasculature in the same blast-exposed animal (coll IV, red; GFAP, green). c, d Loss of collagen IV immunostaining in small vessels associated with hypothalamic enlarged paravascular spaces. GFAP ${ }^{+}$perivascular astrocytic endfeet remained attached (GFAP, green, arrows). e Loss of collagen type IV in the arterial adventitia in regions with enlarged paravascular spaces (a-SMA, green). Arrows in e show arterial regions devoid of collagen IV (red) around a vascular stricture $\left(^{*}\right.$ ). Also note the loss of smooth muscle (a-SMA ${ }^{+}$, green) around the stricture. $\mathbf{f}$ Loss of adventitial collagen IV (arrows) in hippocampal arterioles (coll IV, red; a-SMA, green). DAPI, blue. Scale bar, $140 \mu \mathrm{m}$ a; $20 \mu \mathrm{m}$ for b-f

cells) [23, 87]. The adventitia is lined with astrocytic endfeet and serves as an anchor for nerve endings [36, 87, 129]. Each layer contributes in unique ways to mantaining vascular homeostasis and regulating vascular responses to environmental conditions, stress or injury [129]. The adventitia is the principal vascular injury sensing tissue in which local fibroblasts (mesenchymal progenitors) play a central role in vascular remodeling by modulating smooth muscle cells and interacting with endothelial cells through soluble factors [53, 87, 129]. Adventitial fibroblasts produce and organize elements of the ECM (fibrillar collagens type I and II, collagen type IV, fibronectin, tenascin, osteopontin, thrombospondin), which provide mechanical strength to the vessel $[108,127,129]$. They are also involved in vascular remodeling via the regulation of matrix metalloprotease degradation of the ECM through TIMPs [18, $78,129,144]$.

Acute increases in cerebral MMP-9 associated with blood-brain barrier disruptions have been observed 2-3 h to 1 day post-blast exposure [66]. Blast-induced brain injury, as well as other forms of traumatic brain injury, have shown biphasic patterns of blood-brain barrier disruptions, where the barrier re-establishes itself after the acute phase and reopens at a later point in time $[9,11,55,66,81,106,119]$. Our results show that blast injury induces a chronic remodelling of the arteriolar laminae as evidenced by increased levels of metalloproteinases MMP-2 and MMP-9 observed at 6 weeks post-blast as well as decreased levels of 


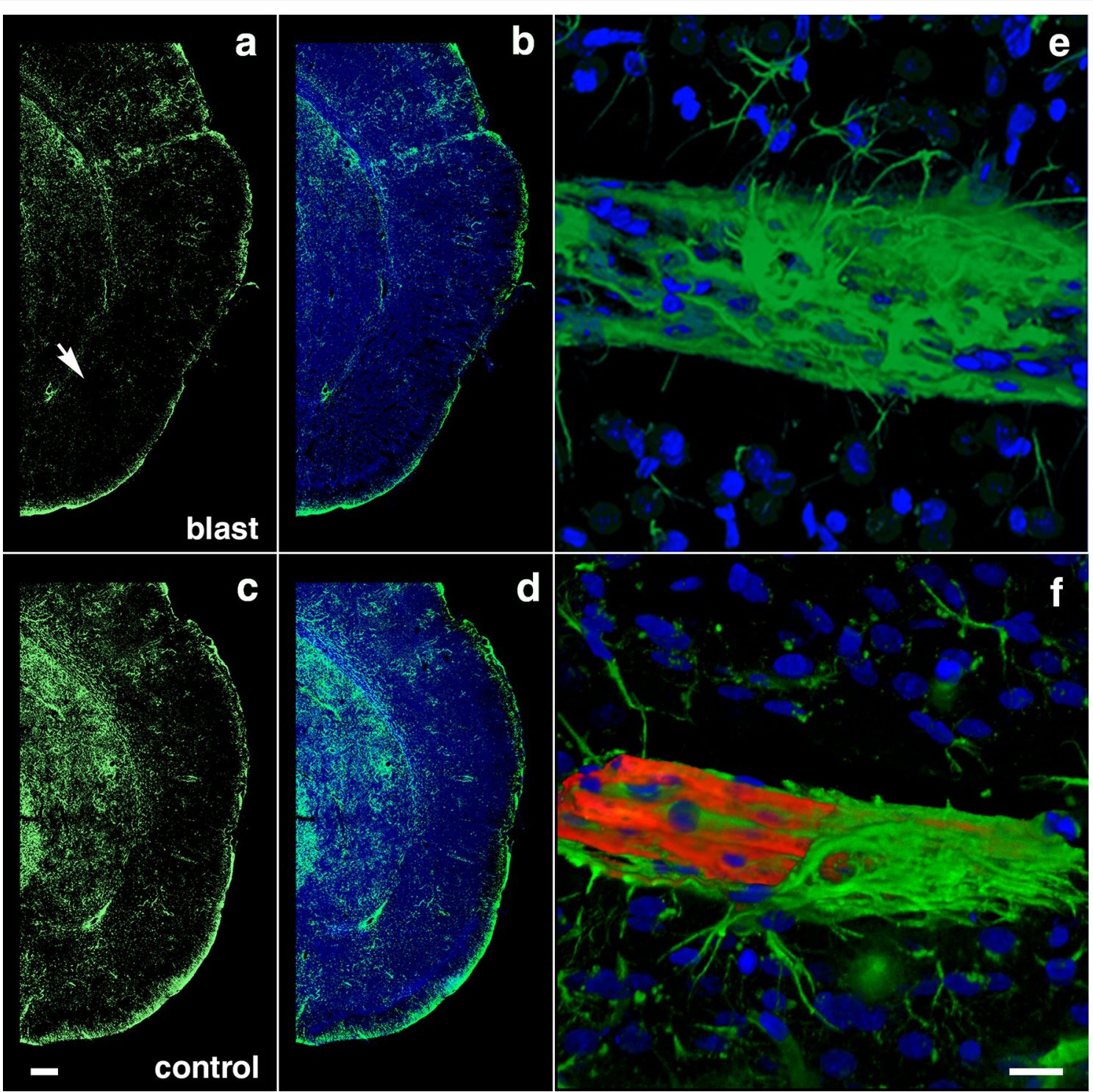

Fig. 10 Reduced GFAP immunoreactivity in brain regions with enlarged paravascular spaces of blast-exposed rats at 13 months post-blast exposure. Coronal brain sections from blast-exposed $\mathbf{a}, \mathbf{b}$ and control $\mathbf{c}$, $\mathbf{d}$ rats were immunostained with antibodies against GFAP (green) and counterstained with DAPI (blue in merged images, $\mathbf{b}, \mathbf{d}$ ). GFAP immunoreactivity was greatly reduced in the thalamus and hypothalamus of blast-exposed animals harboring enlarged paravascular spaces (arrow in a). e, f Abnormally high perivascular astrocytic density in a few large vessels in the entorhinal cortex of blast-exposed rats. Section in e was single-stained with GFAP (green) and $\mathbf{f}$ was double-stained for GFAP (green) and collagen type IV without protease pre-treatment (red,). DAPI (blue). Panel $\mathbf{f}$ also shows GFAP ${ }^{+}$(green) cells under the adventitial collagen type IV (red). Scale bars, $320 \mu \mathrm{m} \mathbf{a}, \mathbf{b}, \mathbf{d}, \mathbf{e} ; 16 \mu \mathrm{m} \mathbf{c}, \mathbf{f}$

vascular collagen type IV in the affected vasculature at 13 months post-exposure.

Matrix metalloproteases are responsible for degradation of ECM components, including basement membrane collagen, interstitial collagen, fibronectin and various proteoglycans [129]. Smooth muscle cells produce metalloproteases [62, 88, 93]. Vascular infiltrating inflammatory cells, endothelial cells and fibroblasts are also known to produce metalloproteases [79]. Excessive expression of vascular MMP-2 and MMP-9 gelatinase activity decreases the elasticity and modifies the structure and mechanical properties of the vessel by degrading the elastic and collagenous fibers of the tunica media and adventitia in addition to the basal lamina.

Acutely, activation of metalloproteases induces vasoconstriction via the transactivation of epidermal growth factor receptors and the production of reactive oxygen species (ROS) [51, 87, 88, 94]. Chronically, 


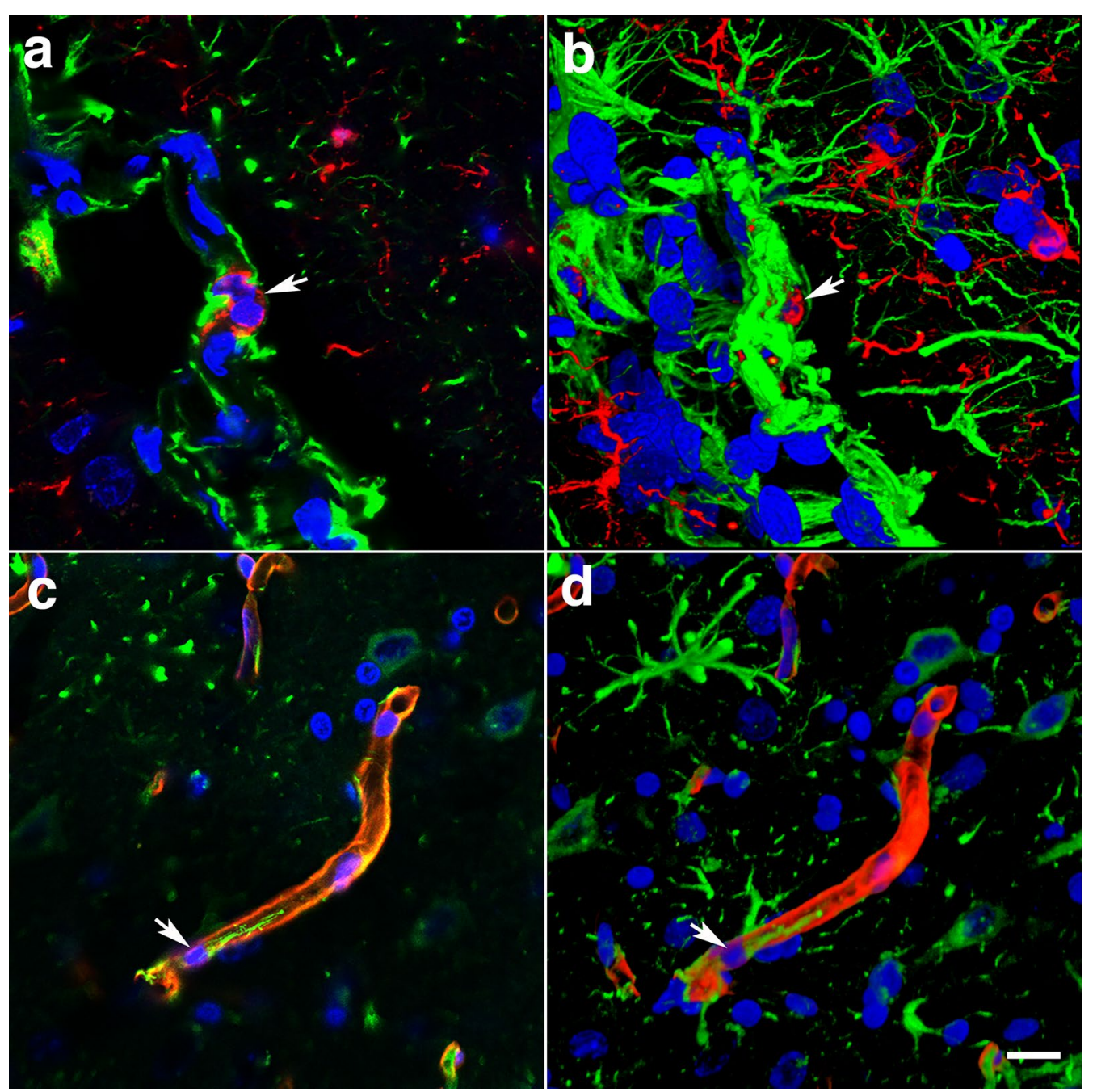

Fig. $11 \mathrm{Microglia/macrophage} \mathrm{and} \mathrm{astrocytic} \mathrm{cells} \mathrm{in} \mathrm{the} \mathrm{vascular} \mathrm{lumen.} \mathrm{a} \mathrm{Confocal} \mathrm{optical} \mathrm{section}(0.56 \mu \mathrm{m})$ showing an Iba ${ }^{+}$microglia/ macrophage (arrow) inside the vascular lumen (Iba 1, red; GFAP, green). Panel $\mathbf{c}$ shows an astrocytic cell (arrow) and its process inside the lumen of a small vessel (GFAP, green; coll IV, red). Panels $\mathbf{b}$ and $\mathbf{d}$ correspond to three-dimensional stack reconstructions of the fields shown in panels $\mathbf{a}$ and $\mathbf{c}$. DAPI, blue. Scale bar, $15 \mu \mathrm{m}$ for $\mathbf{a}, \mathbf{b} ; 20 \mu \mathrm{m}$ for $\mathbf{c}, \mathbf{d}$

metalloproteases and ROS can induce remodeling processes in response to prolonged vasoconstriction [87, 88]. The observed thickened basal and elastic laminae in the cerebral vasculature of blast-exposed animals could be a reflection of this phenomenon (Fig. 14). Altogether, these alterations affecting the composition and physical characteristics of the basal membrane associated with the arterial vascular smooth muscle would predict hypofunctionality of ISF flow via the IPAD system[5]. The presence of extravascular IgG also attests to vascular fragility resulting in leakage, which in turn may trigger more neuroinflammation and carry proteases involved in ECM degradation into the CNS from the periphery.

\section{Chronic astrocytic degeneration}

Astrocytes are multifunctional brain cells that provide ionic and trophic support to neurons, regulate intracerebral circulation and have an important role in cognitive functioning [13, 72, 77, 85, 86, 92, 96, 135]. Perivascular astrocytic degeneration is visible at $72 \mathrm{~h}$ through 6 weeks post-blast with no overt alterations in neuronal somatodendritc morphology [40, 81]. Similarly, decreased levels of GFAP have been observed in rats $24 \mathrm{~h}$ post-blast exposure [117]. Blast exposure induces epigenetic alterations with decreases in astrocytic histone acetylation and altered gene expression [8]. 


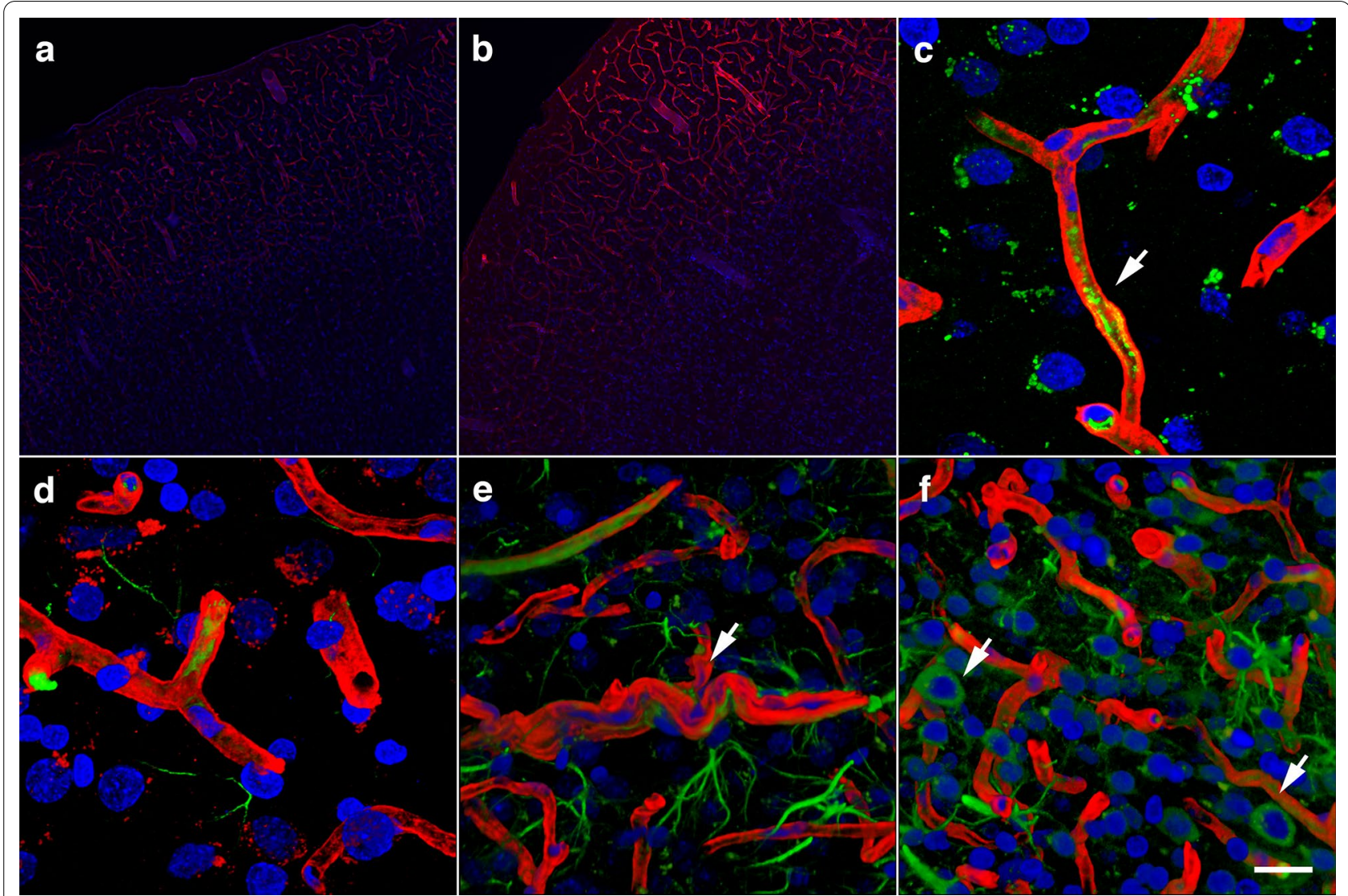

Fig. 12 Chronic proteolysis of collagen type IV in the brains of blast-exposed rats. Unmasking collagen type IV epitopes in the vasculature of normal mature adult rodent brain for immunohistochemical detection requires protease pretreatment [37]. Shown are micrographs of collagen type IV-immunostained sections of brains of control and blast-exposed rats at 13 months post-blast exposure without protease treatment. $\mathbf{a}, \mathbf{b}$, Vasculature in the somatosensory cortex of control $\mathbf{a}$ and blast-exposed $\mathbf{b}$ rats immunostained for collagen type IV (red). Note the absence of collagen IV staining in the section from the control rat a. c A small vessel immunostained for collagen type IV (red) showing MMP-9 vescicles (green, arrow). $\mathbf{d}$-f Collagen type IV (red) and GFAP (green) immunoreactivity of the somatosensory cortical vasculature of blast-exposed rats in the absence of protease pretreatment. Note the tortuosity of the vessel shown in e and the neuronal expression of GFAP (arrows) in this region $\mathbf{f}$. DAPI, blue. Scale bar, $140 \mu \mathrm{m}$ for $\mathbf{a}, \mathbf{b} ; 20 \mu \mathrm{m}$ for $\mathbf{c}-\mathbf{f}$

In our rat model, late chronic vascular alterations induced by blast exposures were previously documented at 6-10 months following exposure [32, 38-40]. In the present study at 13 months post-blast, astrocytic degeneration and perivascular apoptosis was observed to be associated with enlarged paravascular spaces and dissociation of the vasculature from the parenchymal neuropil. Perivascular astrocytic endfeet from the blast-exposed animals were swollen with degenerating organelles, including elongated and swollen mitochondria and cellular debris. It is known that mitochondrial swelling causes rupture of the outer membrane and release of pro-apoptotic proteins such as cytochrome $\mathrm{c}$ into the cytosol [103]. Immunohistochemical analyses and confocal microscopic observations showed evidence for phagocytosis of perivascular astrocytes by activated microglia (Figs. 16, 17).
At advanced degenerative stages, the vasculature was dislocated from the parenchyma as bridging perivascular astrocytes were lost, suggesting that blast induces chronic perivascular astrocytic degeneration. Several studies have documented astrogliosis following blast injury $[8,26,27$, $32,43,65,98]$. In addition, a distinct type of glial scar and cortical perivascular astrocytosis have been described in humans exposed to blast injury [122]. Our study shows widespread, blast-induced loss of perivascular astrocytes. At 13 months post-exposure, loss of perivascular astrocytic coverage was clearly visible in GFAP- and collagen IV-immunolabeled sections and confirmed by electron microscopic observations. The loss of perivascular astrocytes would affect CSF flow through the glymphatic system. The dramatic enlargement of the paravascular space associated with perivascular astrocytic loss in major regions of the brain at 13 months post-blast may also 

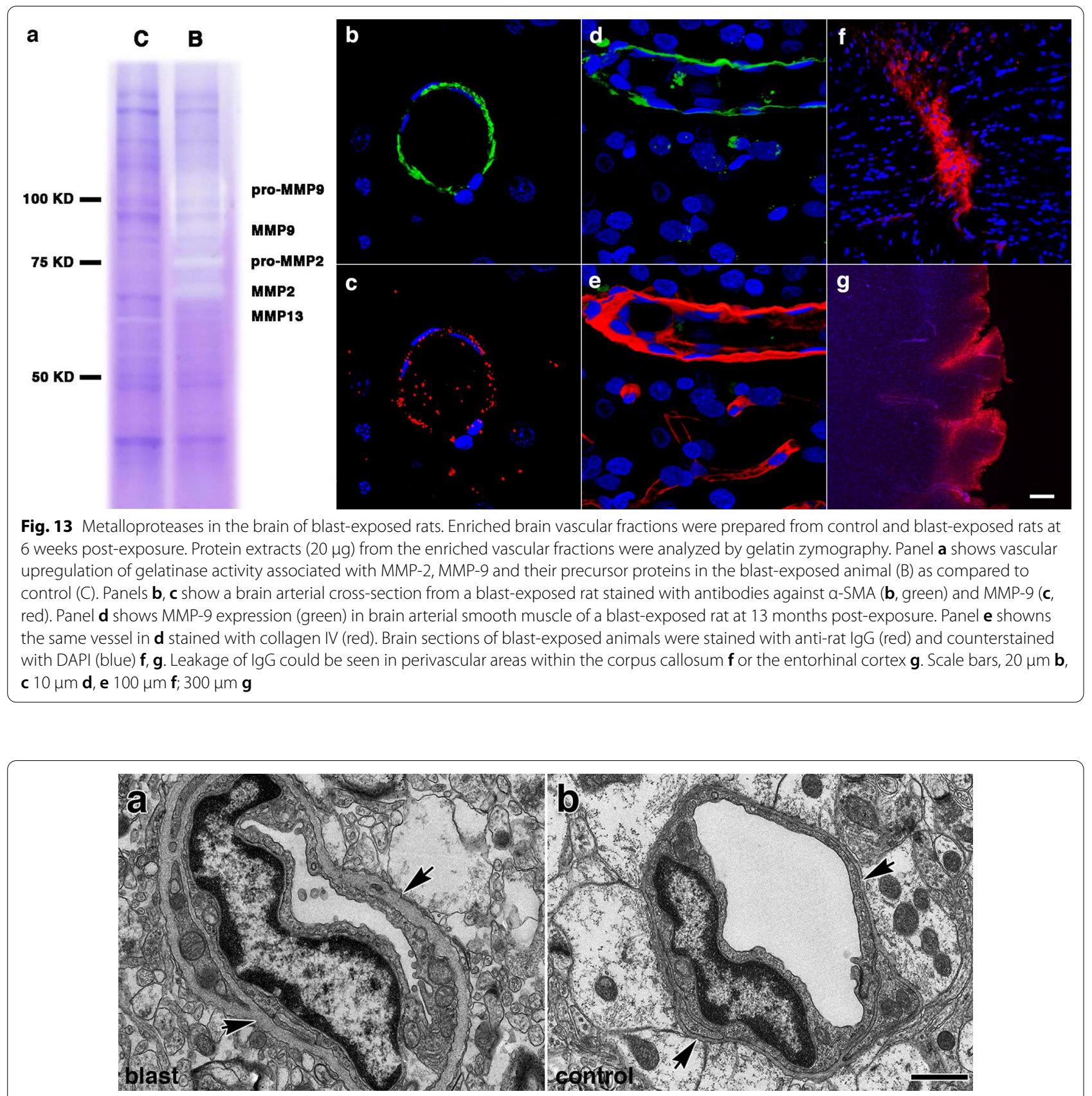

Fig. 14 Blast-induced thickening of the basal lamina surrounding the brain vasculature at 13 months post-blast exposure. Electron micrographs of small vessels in the frontal cortex of blast-exposed $\mathbf{a}$ and control animal $\mathbf{b}$. Note that in the vessel from the blast-exposed animal a compared to control $\mathbf{b}$, there is enhanced thickness of the vascular basal lamina (arrows), a reduction of luminal space, swelling of the endothelial nucleus, and reduction of astrocytic endfeet contacts. The endothelial membrane in contact with the lumen is distorted and has an irregular "wavy" pattern. Also, note that in panel a the cytosol of the remaining astrocytes lack the ribosomal density present in the normal astrocytic cytoplasm of control vessels b. Scale bar, $1 \mu \mathrm{m}$

indicate the continuous expansion of initial blast-induced neurovascular disconnections across the vasculature through the mechanical action of heartbeat-induced arterial peristaltic pulsations [68]. Supporting a possible similar effect in humans, Piantino et al. [104] found evidence for enlarged perivascular spaces by MRI in living Iraq/ Afghanistan veterans who had been exposed to blast. These enlarged perivascular spaces (termed PVS burden) had a significant positive correlation with number of blast-induced TBIs [104]. They were also associated 


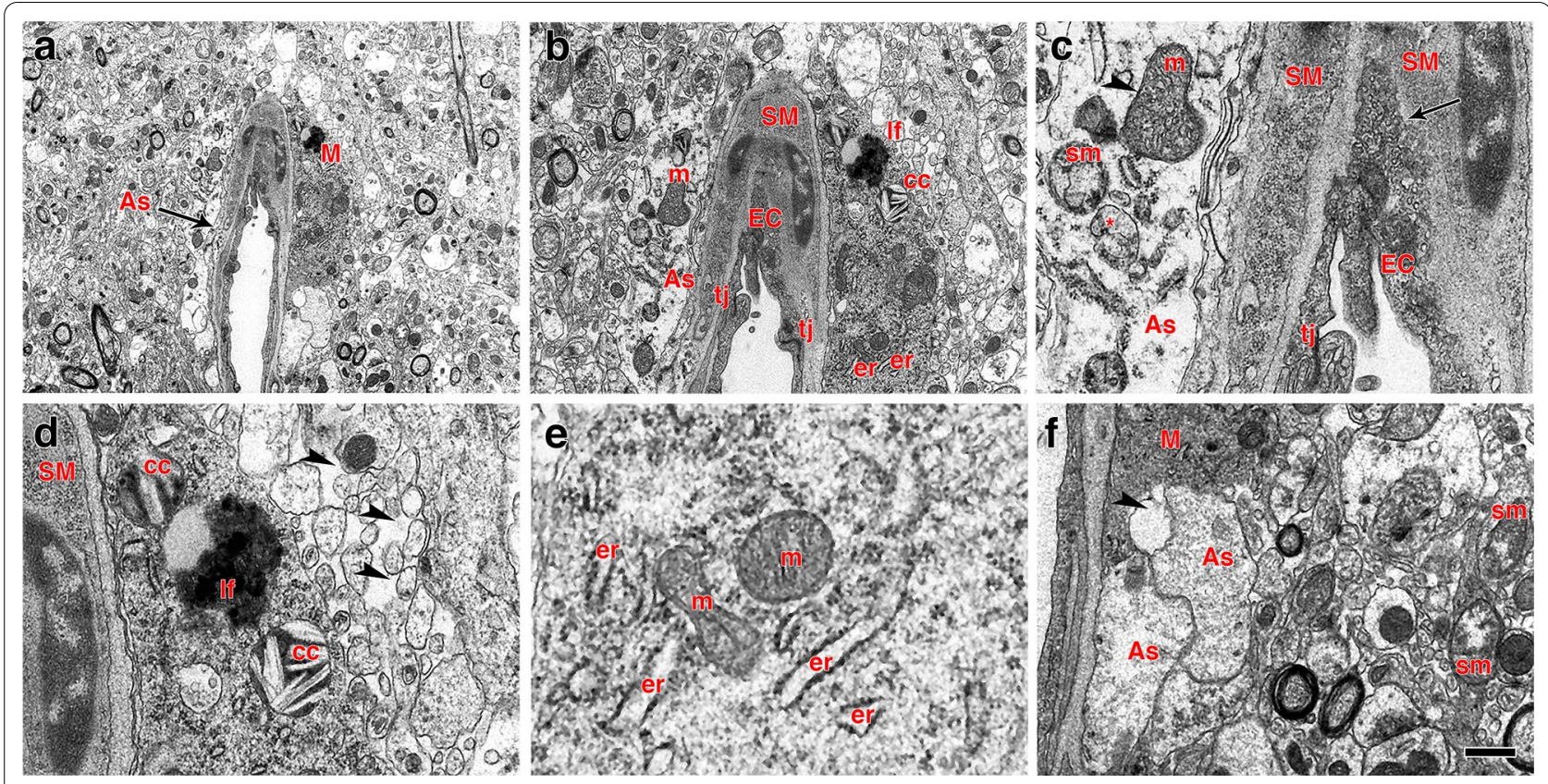

Fig. 15 Perivascular microglia and vascular degeneration. a-f Electron micrographs of a perivascular microglial cell (M) adjoining a degenerating arteriole. Perivascular astrocytic feet (As, arrow) exhibit lack of ribosomes and altered mitochondria including swollen mitochondria (sm, panel c), mitochondria with disorganized cristae (arrowhead in c) and completely dystrophic mitochondria (* in c). The intimal endothelial cells (EC, panels a-c) are partially dislodged from the basal lamina with free processes protruding into the lumen. The abnormal endothelial cells exhibit a high density of small osmiophilic vescicles. Note the lack of definition of the basal lamina between endothelial and smooth muscle (SM) cells (arrow in c). The perivascular microglial cell $\mathbf{a}, \mathbf{b}, \mathbf{d}-\mathbf{f}$ shows lysosomal cholesterol crystals (cc in panels $\mathbf{b}$ and $\mathbf{d}$ ), a lipofuscin body (If in panels $\mathbf{b}$ and $\mathbf{c}$ ) and enlarged endoplasmic reticulum cisternae (er) characteristic of the "dark" microglial phenotype e. Arrowheads in panel $\mathbf{d}$ show abnormal enhanced interstitial spaces in the neuropil neighboring the perivascular microglial cell. Arrowhead in panel $\mathbf{f}$ indicates remnants of an astrocytic foot process next to a microglial process. m, mitochondria; tj, tight junction. Scale bars, $2.5 \mu \mathrm{m} \mathbf{a} ; 1 \mu \mathrm{m} \mathbf{b} ; 0.2 \mu \mathrm{m} \mathbf{c} ; 0.4 \mu \mathrm{m} \mathbf{d}, \mathbf{e} ; 0.8 \mu \mathrm{m} \mathbf{f}$

with poor sleep and persistent post-concussive symptom severity. Thus, multiple lines of evidence in animals and humans support the notion that the perivascular space is disturbed and enlarged after blast injury.

One curious observation is the presence of intraluminal astrocytic processes within blood vessels (Fig. 10). This phenonenon has been repeatedly observed in this and previous studies [40]. We suspect that the intraluminal astrocytic processes appear in hypofunctional damaged vessels into which astrocytic cells and processes could extend. Based on earlier work by six weeks after blast exposure, there is loss of GFAP in isolated vascular fractions with swelling and degeneration of astrocytic endfeet, creating an effective gliovascular disconnection [40]. However, vascular fractions from animals taken at 8 months post-blast exposure show normal levels of GFAP, suggesting some attempt at restablishing astrocytic perivascular connections [40] although the connections remain highly abnormal (Fig. 6). The unusual accumulation of perivascular astrocytes in some affected vein-like vessels at 13 months post-exposure further supports a late-onset astrocytic recovery, that although abnormal is occuring in some vessels (Fig. 10).
Reductions in cerebral blood flow result in release of vasoactive metabolites that induce vasodilation [23]. Blood flow is also controlled by hypercapnia and hypoxia, which induce dilation of cerebral arteries and arterioles, resulting in increased blood flow. On the other hand, hypocapnia causes vasoconstriction and decreased blood flow. Arterial autoregulation of blood flow is further maintained by contraction of arterial smooth muscle, which is also disturbed in this model [40]. Unlike pial arteries and arterioles, parenchymal arterioles are in close association with astrocytes and to a lesser extent with neurons [23]. Cortical vessels are innervated from subcortical brain regions with the majority of neural varicosities targeting the astrocytic endfeet surrounding arterioles [74].

Although direct signaling from neurons to blood vessels contributes to the regulation of cerebral blood flow, astrocytes play an important role in mediating neurovascular coupling $[7,60,70]$. Glutamate released from neurons acts on astrocytic metabotropic glutamate receptors to evoke $\mathrm{Ca}^{2+}$-dependent release of vasoactive metabolites of arachidonic acid from astrocytic endfeet. These astrocytic mechanisms, regulated 

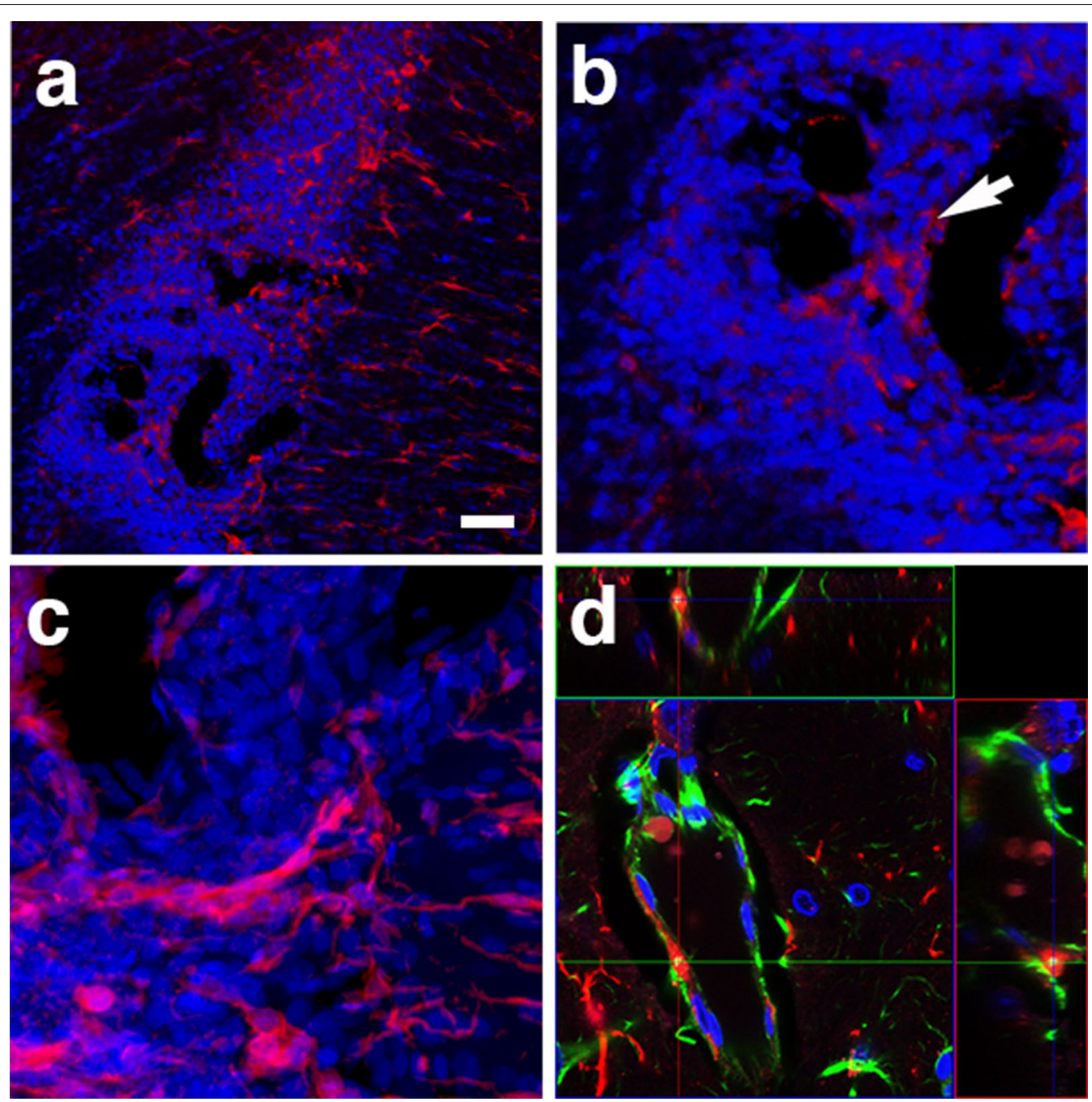

Fig. 16 Perivascular microglia in the brain of blast-exposed animals. Panels a-c show clustered lba $1^{+}$microglia (red, arrow in b) with amoeboid morphology in the perivascular area of a tortuous large vessel (pericallosal artery) in the corpus callosum of a rat brain at 13 months post-blast exposure. Panel $\mathbf{d}$ shows an orthogonal projection of a section through a thalamic artery stained for perivascular astrocytes (GFAP ${ }^{+}$, green) and microglia (Iba $1^{+}$, red). Co-localization of astrocytic GFAP ${ }^{+}$material within a perivascular microglial cell, suggests phagocytic ingestion of perivascular astrocytes by microglia. Scale bar, $100 \mu \mathrm{m} \mathbf{a} ; 40 \mu \mathrm{m}$ b; $20 \mu \mathrm{m} \mathbf{c}, \mathbf{d}$

by $\mathrm{pO}_{2}$ and lactate, induce either vascular dilation via prostaglandin E2 (PGE2) and epoxyeicosatrienoic acids (EETs) or vasoconstriction via 20-hydroxyeicosatetraenoic acid (20-HETE). Under normoxic conditions, astrocytic $\mathrm{Ca}^{2+}$ signaling results in vasodilation. Under hyperoxia, however, vasoconstriction is favored. Release of $\mathrm{K}^{+}$from astrocytic endfeet upon active neuronal stimulation may also contribute to vasodilation [35]. Direct stimulation of astrocytes raises calcium levels in the endfeet to induce the release of $\mathrm{K}^{+}, 20$-HETE and PGE2, which in turn causes dilation of neighboring arterioles $[34,90,146]$.
Neuronal activity may also regulate capillary blood flow through $\mathrm{Ca}^{2+}$-dependent astrocytic mechanisms $[15,89]$ that actively dilate and constrict pericytes in response to vasoactive agents. Pericyte-induced relaxation has been shown to be mediated by activation of PGE2 receptors and by nitric oxide inhibition of the synthesis of the vasoconstrictor 20-HETE [47, 84]. Pericyte degeneration leads to neurovascular uncoupling and limits oxygen supply to the brain [71].

Control of local blood flow to match neuronal demand results, in part, from the release of neurotransmitters that stimulate receptors on smooth muscle, endothelium, pericytes or astrocytes to induce 

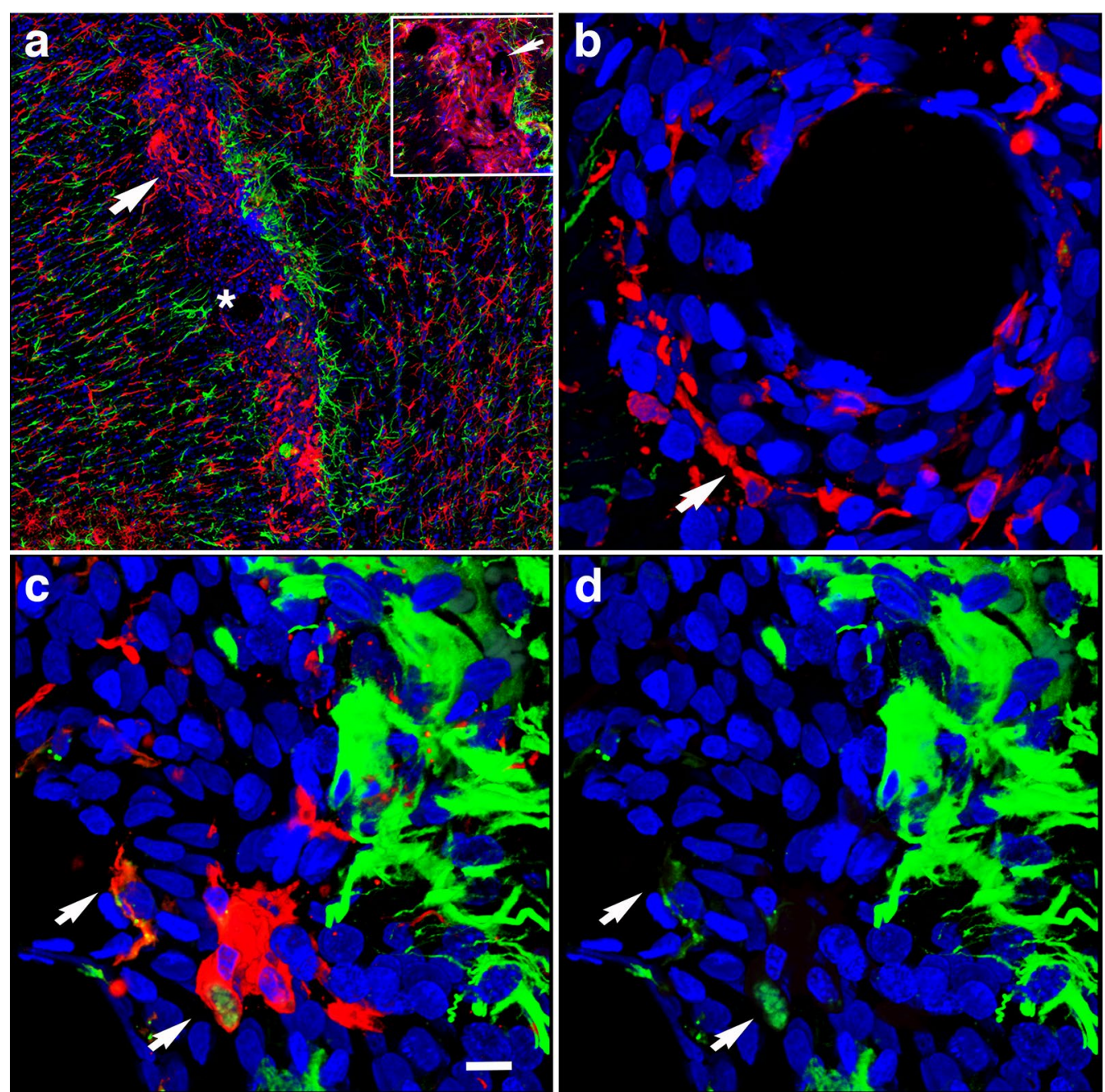

Fig. 17 Perivascular inflammation in the brain of a blast-exposed animal. Panel a shows a brain region with patches of clustered activated microglia (Iba ${ }^{+}$, red) next to a vascularized region in between the deep cerebral white matter, choroid plexus and hippocampal fimbria. Insert in a shows higher magnification of a $0.56 \mu \mathrm{m}$ optical section of the area indicated by arrow. Note the tortuous vessel (arrow). Panel $\mathbf{b}$ denotes higher magnification of an artery (identified by ${ }^{*}$ in panel a) with perivascular activated microglia (lba $1^{+}$, red). Panels $\mathbf{c}, \mathbf{d}$ show GFAP ${ }^{+}$intracellular material (green) in perivascular activated microglia (Iba1, red; GFAP, green). Scale bar, $200 \mu \mathrm{m}$ a; $10 \mu \mathrm{m}$ b-d

constriction or dilation through multiple signaling pathways [7, 23, 29, 48, 49, 58-60, 109, 121]. Therefore, any disruption within the neurovascular unit by blast exposure is likely to result in altered cerebral perfusion. As confirmed here, blast exposure results in structural alterations of the arterial medial smooth muscle layer. In addition to disrupted gliovascular and neurovascular signaling and cerebral circulation, glymphatic flow and IPAD drainage could also be impaired by altered vascular smooth muscle structure $[5,142]$. In the human context failure of the glymphatic and IPAD systems may translate in the long term to Abeta deposition, development of cerebral amyloid angiopathy (CAA) and Alzheimer's disease [5].
Microglia activation associated with vascular degeneration Microglia are small macrophage-like glial cells of the central nervous system that constitute $\sim 10 \%$ of the cells in the brain $[57,76,107]$. They are in contact with synaptic elements (axon terminals, dendritic spines, astrocytic processes and synaptic clefts) where they maintain homeostasis, remove tissue debris and pathogens, and remodel synapses [16]. Upon neural injury, pathological states or chronic stress, microglia are activated and undergo morphological, proliferative and physiological changes that increase phagocytic activity and secretion of proinflammatory molecules [107]. This results in propagation of neuroinflammatory and neurodegenerative responses. 

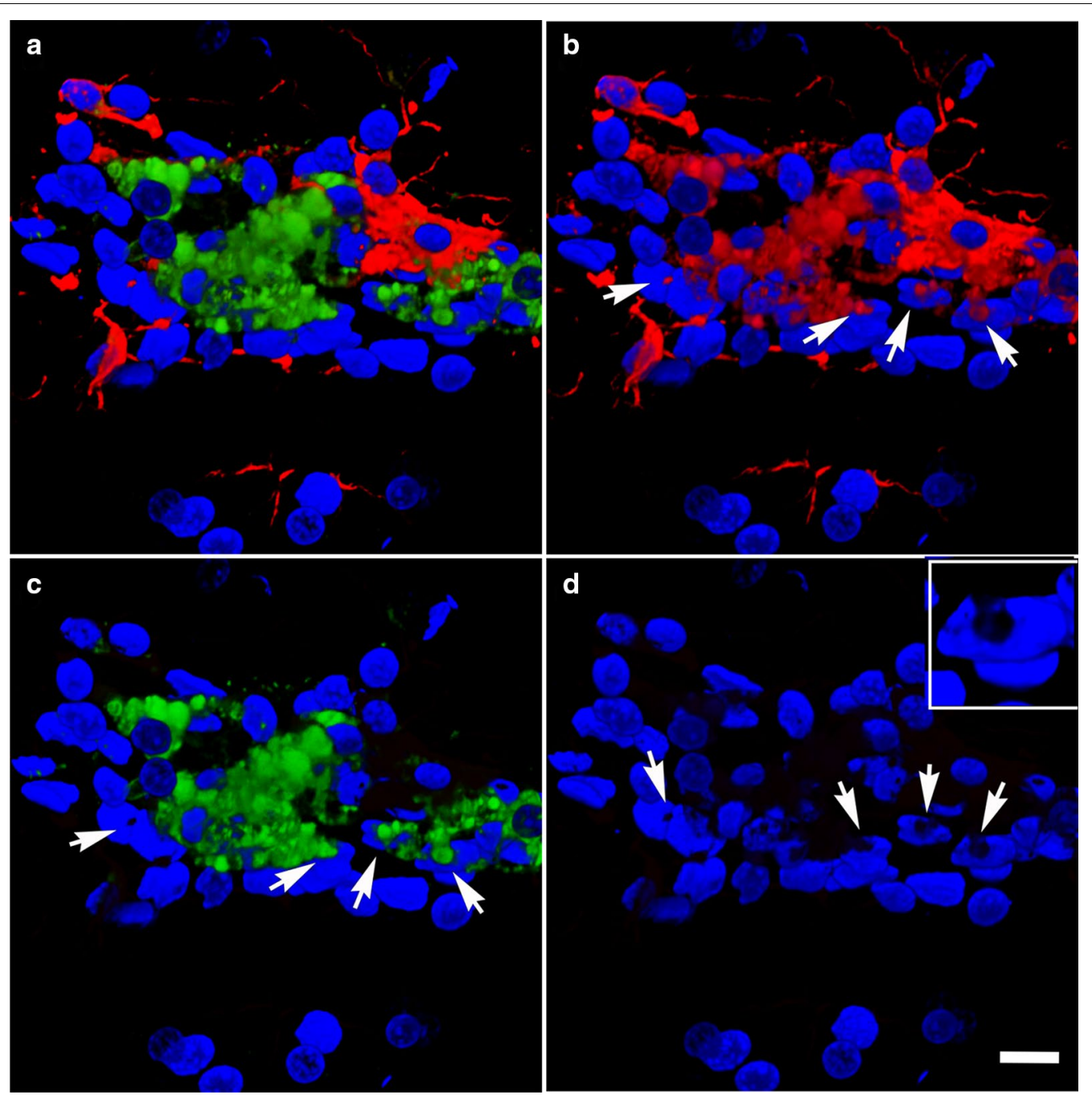

Fig. 18 Perivascular microglial activation. MHCll expression is associated with microglial activation. Shown are perivascular amoeboid Iba ${ }^{+}$ microglia (red). Some microglia are also expressing MHCII (green) and extend transcellular processes across the nuclei of Lochkern-like cells. $\mathbf{a}$, Merged images, b Iba1 staining (red); c MHCIl staining (green); and d DAPI staining (blue). Insert in d shows an enhanced view of a Lochkern-like cell. Arrows indicate the location of the Lochkern-like cells with hollow nuclei and the transcellular microglial processes $\left(\mathrm{Iba} 1^{+} \mathrm{and} \mathrm{MHCII}^{+}\right.$). Cellular blebs indicate that $\mathrm{MHCII}^{+}$microglia appear to be undergoing apoptosis. Scale bar, $15 \mu \mathrm{m}$ a-d; $5 \mu \mathrm{m}$ insert in $\mathbf{d}$

Microglial morphologies are associated with different states of activation and include ramified, primed, reactive, and ameboid microglia (Types $1-4$, respectively) [69, 73, 120, 124, 128, 139]. Functional microglial activation states include the pro-inflammatory M1 activation associated with enhanced neurotoxicity and ECM damage, anti-inflammatory and neuroprotective M2a activation that induces phagocytosis, and the microglia-deactivating M2c activation involved in wound healing [143].

In a previous study we found no morphological or biochemical evidence of inflammation in the brains of rats 6 weeks post-blast exposure [41]. However, neuroinflammation was reported in an animal 16 weeks post-blast exposure with evidence of vascular rupture [41]. In the present study we present evidence that rats 13 months post-blast exposure exhibited neuroinflammation associated with vascular alterations including the presence of perivascular activated $\mathrm{MHCII}^{+}$microglia. Expression of the major histocompatibility class II (MHCII) in microglia is linked to the inflammatory response in neurodegenerative diseases [118]. Our results indicate that this vascular-associated neuroinflammation develops after the subacute phase ( 6 weeks post-blast) into the chronic stage. In association with degenerating perivascular astrocytic endfeet, we also identified microglia with 


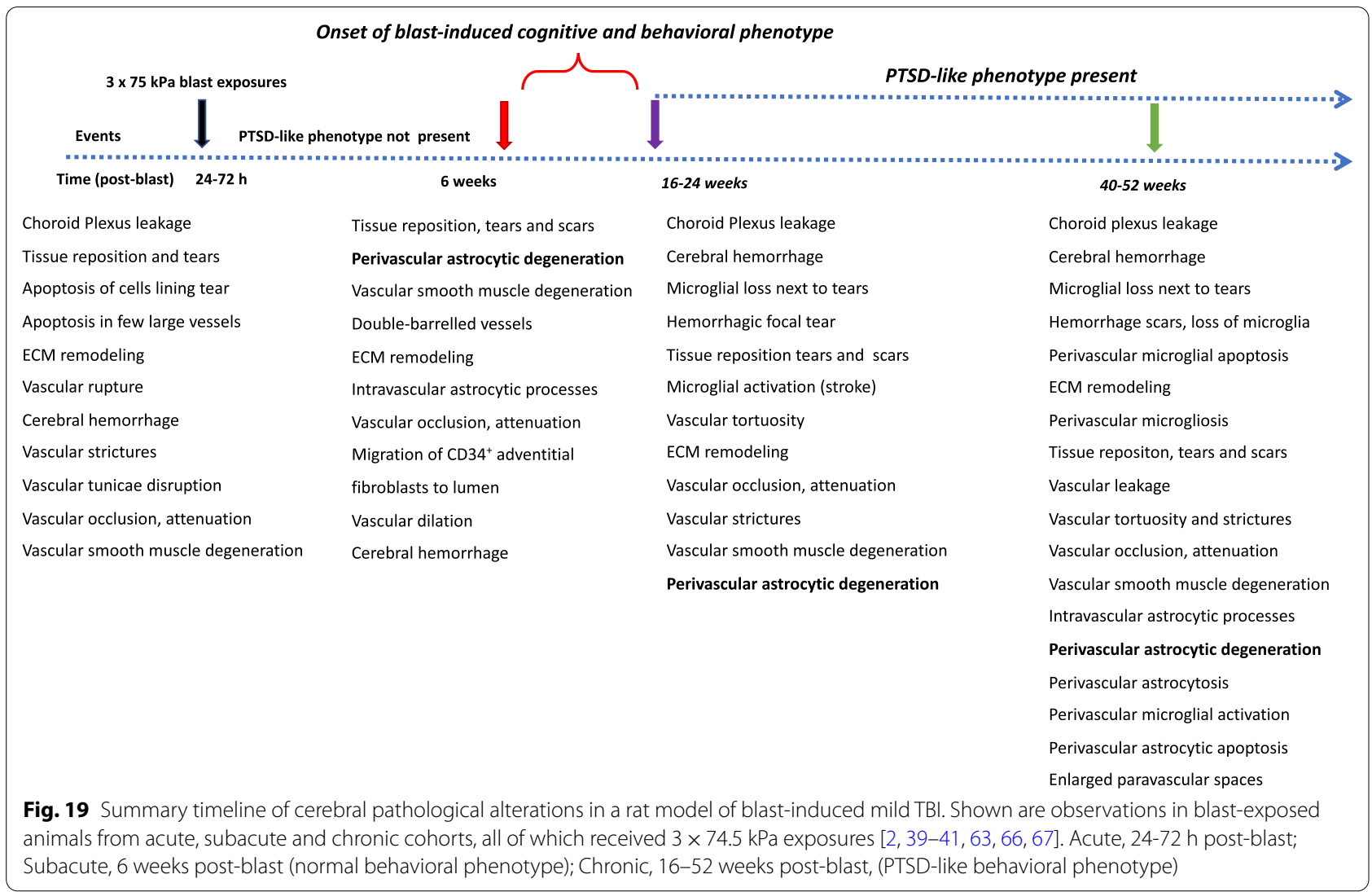

dilated endoplasmic reticulum, mitochondrial alterations and cholesterol crystals in lysosomal vacuoles. Cholesterol crystals have been shown to induce destabilization of lysosomes, causing leakage of the protease cathepsin $B$, leading to activation of the innate NLRP3 inflammasome complex, resulting in caspase-1-mediated activation and secretion of proinflammatory cytokines [44, 110]. Following blast-induced vascular fragility and leakage, cholesterol-rich debris (including myelin) may be engulfed by microglia and infiltrating macrophages into phagosomes, which fuse with lysosomes to form phagolysosomes. Compromised reverse cholesterol transport, which normally mediates efflux of cholesterol will result in cholesterol overload, leading to formation of lysosomal membrane-damaging cholesterol crystals, release of lysosomal enzymes, inflammasome activation and cytokine secretion [80].

It has been shown that local inflammation associated with vascular degeneration may be due to elevated permeability of the blood-brain barrier. This results in clustering of activated microglia which contact the affected vasculature $[16,24,46,52,126]$. The dilated endoplasmic reticulum identifies these cells as dark microglia $[16$, 126].
Interestingly, rare activated microglia neighboring blood vessels contained cells with hollow nuclei (Lochkern-like cells $[12,105])$ through which microglial processes seemed to transverse. Although the significance of this unusual observation remains unknown, transcellular migration of microglia has been extensively described in the retina after neuronal damage $[64,95,111,137]$. This process is involved in the rapid recruitment and migration of immune and inflammatory cells to inflammatory sites without the loss of barrier integrity. Microglial transcellular and paracellular transport within the neurovascular unit [52] may be increased by the chronic vascular degenerative events induced by blast exposure.

Microglia are also known to have a critical role in vascular repair and maintainance of vascular integrity in the central nervous system [46]. Chronic mild hypoxia in the spinal cord induces transient vascular leakage associated with activated microglia clustering next to the affected vasculature, particularly in the white matter. Microglial depletion in this system leads to exaggerated vascular leakage associated with astrocyte-vascular uncoupling and loss of endothelial tight junction proteins. Microglial vascular repair depends on fibrinogen-Mac1 receptor interactions [46]. Similarly, in our rat model of blast-induced traumatic brain injury, at 13 months 
post-exposure we observed clustered activated microglia next to affected vasculature in the brain white matter. Some of these cells exhibited intracellular $\mathrm{GFAP}^{+}$ material as evidence for microglial phagocytosis of astrocytes. TUNEL analysis showed perivascular apoptosis of astrocytes and astrocytic-associated microglia. The brains of blast-exposed animals at this late time point also presented vascular leakage as evidenced by the presence of extravascular IgG. Microglial repair function may prevent further major leakage. Apoptotic loss of perivascular microglia may result in loss of vascular repair and increased vascular fragility.

\section{Conclusions}

Our results show that blast exposure affects cellular interactions within the neurovascular unit and that astrocytes play a central role bridging the vasculature and the parenchymal neuropil. The cognitive and behavioral alterations that develop following blast exposure in both humans and animal models [30, 31, 33, 83, 99, 102] may be caused by the resulting disruptions in blood circulation, glymphatic CSF flow and intramural periarterial ISF drainage that develop as the result of altered neurogliovascular structure, astrocytic degeneration, vascular remodeling, vascular smooth muscle degeneration, vascular fragility, vascular leakage and neuroinflammation. Furthermore, impaired microglial function may impede vascular repair and accelerate the neurovascular degenerative processes associated with blast-induced traumatic brain injury.

\begin{abstract}
Acknowledgements
We sincerely thank the anonymous reviewers whose constructive suggestions improved this manuscript considerably. We also thank Dr. Virginia Lee for generously providing the rat anti-GFAP antibody. The work described in this paper was supported by the Department of Veterans Affairs, Veterans Health Administration, Rehabilitation Research and Development Service Awards 1101BX0004067-01 (GE), I01RX002660-01 (GE), 1 I21 RX003459-01 (MAGS), 1 I 21 RX002069-01 (MAGS) and 1 I21 RX002876-01 (MAGS); by the Department of Defense work unit number 0000B999.0000.000.A1503, the Alzheimer's Drug Discovery Foundation (SG) and by NIA P50 AG011508 and P30 AG066514 to Mary Sano (SG, PRH). MAGS, RDG, DP, GMP, GE, SG, RA, UK, DGC and STA are employees of the U.S. Government. This work was prepared as part of their official duties. Title 17 U.S.C. \$105 provides that 'Copyright protection under this title is not available for any work of the United States Government'.Title 17 U.S.C. \$101 defines a U.S. Government work as a work prepared by a military service member or employee of the U.S. Government as part of that person's official duties. The views expressed in this article are those of the authors and do not necessarily reflect the official policy or position of the Department of the Navy, Department of Defense, Department of Veterans Affairs, nor the U.S. Government.
\end{abstract}

\section{Authors' contributions}

MAGS, RDG, DP, GPG, SG, WGJ, DC, SG, SJT, PRH and GAE: design of experiments, analysis and interpretation of data, design of neuropathological characterization, biochemical assays and manuscript writing; DP and GMP: execution of neuropathological characterization; GPG and GMP: behavior tests; WGJ and AS: electron microscopy; SH and BA: micro-CT scanning; TT and SJT: morphological and automated quantitative analyses of the cerebral vasculature; STA, RMM, RA and UK: design and conduct of blast experiments, interpretation of data and manuscript writing. All authors read and approved the manuscript.

\section{Availability of data and materials}

The datasets generated during and/or analysed during the current study are available from the corresponding author on reasonable request.

\section{Declarations}

\section{Competing interests}

The authors declare that they have no competing interests.

\section{Author details}

${ }^{1}$ General Medical Research Service, James J. Peters Department of Veterans Affairs Medical Center, 130 West Kingsbridge Road, Bronx, NY 10468, USA. ${ }^{2}$ Department of Psychiatry, Icahn School of Medicine at Mount Sinai, One Gustave Levy Place, New York, NY 10029, USA. ${ }^{3}$ Friedman Brain Institute, Icahn School of Medicine at Mount Sinai, New York, NY 10029, USA. ${ }^{4}$ Research and Development Service, James J. Peters Department of Veterans Affairs Medical Center, 130 West Kingsbridge Road, Bronx, NY 10468, USA. ${ }^{5}$ Department of Neurology, Icahn School of Medicine at Mount Sinai, One Gustave Levy Place, New York, NY 10029, USA. ${ }^{6}$ Nash Family Department of Neuroscience, Icahn School of Medicine at Mount Sinai, New York, NY 10029, USA. ${ }^{7}$ Department of Neurotrauma, Operational and Undersea Medicine Directorate, Naval Medical Research Center, 503 Robert Grant Avenue, Silver Spring, MD 20910, USA. ${ }^{8}$ The Henry M. Jackson Foundation for the Advancement of Military Medicine Inc, Bethesda, MD, USA. ${ }^{9}$ Micro Photonics, Inc, 1550 Pond Road, Suite 110, Allentown, PA 18104, USA. ${ }^{10}$ MBF Bioscience LLC, 185 Allen Brook Lane, Williston, VT 05495, USA. ${ }^{11}$ Geriatric Research Education and Clinical Center, VA Puget Sound Health Care System, 1660 S Columbian Way, Seattle, WA 98108, USA. ${ }^{12}$ Department of Medicine, University of Washington, 1959 NE Pacific St, Seattle, WA 98195, USA. ${ }^{13}$ Mount Sinai Alzheimer's Disease Research Center and the Ronald M. Loeb Center for Alzheimer's Disease, Icahn School of Medicine at Mount Sinai, New York, NY 10029, USA. ${ }^{14}$ NFL Neurological Care Center, Icahn School of Medicine at Mount Sinai, New York, NY 10029, USA. ${ }^{15}$ Department of Geriatrics and Palliative Care, Icahn School of Medicine at Mount Sinai, New York, NY 10029, USA. ${ }^{16}$ Neurology Service, James J. Peters Department of Veterans Affairs Medical Center, 130 West Kingsbridge Road, Bronx, NY 10468, USA.

Received: 25 May 2021 Accepted: 29 September 2021

Published online: 15 October 2021

\section{References}

1. Abbott NJ (2004) Evidence for bulk flow of brain interstitial fluid: significance for physiology and pathology. Neurochem Int 45:545-552. https://doi.org/10.1016/j.neuint.2003.11.006

2. Abutarboush R, Gu M, Kawoos U, Mullah SH, Chen Y, Goodrich SY, Lashof-Sullivan M, McCarron RM, Statz JK, Bell RS et al (2019) Exposure to blast overpressure impairs cerebral microvascular responses and alters vascular and astrocytic structure. J Neurotrauma 36:3138-3157. https://doi.org/10.1089/neu.2019.6423

3. Ackermans NL, Varghese M, Wicinski B, Torres J, De Gasperi R, Pryor D, Elder GA, Gama Sosa MA, Reidenberg JS, Williams TM et al (2021) Unconventional animal models for traumatic brain injury and chronic traumatic encephalopathy. J Neurosci Res. https://doi.org/10.1002/jnr. 24920

4. Ahlers ST, Vasserman-Stokes E, Shaughness MC, Hall AA, Shear DA, Chavko M, McCarron RM, Stone JR (2012) Assessment of the effects of acute and repeated exposure to blast overpressure in rodents: toward a greater understanding of blast and the potential ramifications for injury in humans exposed to blast. Front Neurol 3:32

5. Aldea R, Weller RO, Wilcock DM, Carare RO, Richardson G (2019) Cerebrovascular smooth muscle cells as the drivers of intramural periarterial drainage of the brain. Front Aging Neurosci 11:1. https://doi.org/10. 3389/fnagi.2019.00001

6. Alford PW, Dabiri BE, Goss JA, Hemphill MA, Brigham MD, Parker KK (2011) Blast-induced phenotypic switching in cerebral vasospasm. Proc 
Natl Acad Sci U S A 108:12705-12710. https://doi.org/10.1073/pnas. 1105860108

7. Attwell D, Buchan AM, Charpak S, Lauritzen M, Macvicar BA, Newman EA (2010) Glial and neuronal control of brain blood flow. Nature 468:232-243. https://doi.org/10.1038/nature09613

8. Bailey ZS, Grinter MB, VandeVord PJ (2016) Astrocyte reactivity following blast exposure involves aberrant histone acetylation. Front Mol Neurosci 9:64. https://doi.org/10.3389/fnmol.2016.00064

9. Baldwin SA, Fugaccia I, Brown DR, Brown LV, Scheff SW (1996) Bloodbrain barrier breach following cortical contusion in the rat. J Neurosurg 85:476-481. https://doi.org/10.3171/jns.1996.85.3.0476

10. Ballabh P, Braun A, Nedergaard M (2004) The blood-brain barrier: an overview: structure, regulation, and clinical implications. Neurobiol Dis 16:1-13. https://doi.org/10.1016/j.nbd.2003.12.016

11. Baskaya MK, Rao AM, Dogan A, Donaldson D, Dempsey RJ (1997) The biphasic opening of the blood-brain barrier in the cortex and hippocampus after traumatic brain injury in rats. Neurosci Lett 226:33-36. https://doi.org/10.1016/s0304-3940(97)00239-5

12. Bavle RM (2016) Nuclear vacuolization: Giant Lochkern-like cells. J Oral Maxillofac Pathol 20:339-341. https://doi.org/10.4103/0973-029X. 190895

13. Ben Menachem-Zidon O, Avital A, Ben-Menahem Y, Goshen I, Kreisel T, Shmueli EM, Segal M, Ben Hur T, Yirmiya R (2011) Astrocytes support hippocampal-dependent memory and long-term potentiation via interleukin-1 signaling. Brain Behav Immun 25:1008-1016. https://doi. org/10.1016/j.bbi.2010.11.007

14. Benveniste H, Liu X, Koundal S, Sanggaard S, Lee H, Wardlaw J (2019) The glymphatic system and waste clearance with brain aging: a review. Gerontology 65:106-119. https://doi.org/10.1159/000490349

15. Biesecker KR, Srienc Al, Shimoda AM, Agarwal A, Bergles DE, Kofuji P, Newman EA (2016) Glial cell calcium signaling mediates capillary regulation of blood flow in the retina. J Neurosci 36:9435-9445. https:// doi.org/10.1523/JNEUROSCI.1782-16.2016

16. Bisht K, Sharma KP, Lecours C, Sanchez MG, El Hajj H, Milior G, OlmosAlonso A, Gomez-Nicola D, Luheshi G, Vallieres L et al (2016) Dark microglia: a new phenotype predominantly associated with pathological states. Glia 64:826-839. https://doi.org/10.1002/glia.22966

17. Blaze J, Choi I, Wang Z, Umali M, Mendelev N, Tschiffely AE, Ahlers ST, Elder GA, Ge Y, Haghighi F (2020) Blast-related mild TBI alters anxietylike behavior and transcriptional signatures in the rat amygdala. Front Behav Neurosci 14:160. https://doi.org/10.3389/fnbeh.2020.00160

18. Brauer PR (2006) MMPs-role in cardiovascular development and disease. Front Biosci 11:447-478. https://doi.org/10.2741/1810

19. Carare RO, Bernardes-Silva M, Newman TA, Page AM, Nicoll JA, Perry VH, Weller RO (2008) Solutes, but not cells, drain from the brain parenchyma along basement membranes of capillaries and arteries: significance for cerebral amyloid angiopathy and neuroimmunology. Neuropathol Appl Neurobiol 34:131-144. https://doi.org/10.1111/j. 1365-2990.2007.00926.x

20. Chavko M, Koller WA, Prusaczyk WK, McCarron RM (2007) Measurement of blast wave by a miniature fiber optic pressure transducer in the rat brain. J Neurosci Methods 159:277-281

21. Chavko M, Prusaczyk WK, McCarron RM (2006) Lung injury and recovery after exposure to blast overpressure. J Trauma 61:933-942

22. Chavko M, Watanabe T, Adeeb S, Lankasky J, Ahlers S, McCarron R (2011) Transfer of pressure wave through the body and its impact on the brain. In: Nato symposium on a survey of blast injury across a full landscape of military science, city

23. Cipolla MJ (2009) The cerebral circulation. Morgan \& Claypool Life Sciences, San Rafael, CA. Available from: https://www.ncbi.nlm.nih.gov/ books/NBK53081/

24. da Fonseca AC, Matias D, Garcia C, Amaral R, Geraldo LH, Freitas C, Lima FR (2014) The impact of microglial activation on blood-brain barrier in brain diseases. Front Cell Neurosci 8:362. https://doi.org/10.3389/fncel. 2014.00362

25. De Gasperi R, Gama Sosa MA, Kim SH, Steele JW, Shaughness MC, Maudlin-Jeronimo E, Hall AA, Dekosky ST, McCarron RM, Nambiar MP et al (2012) Acute blast injury reduces brain abeta in two rodent species. Front Neurol 3:177. https://doi.org/10.3389/fneur.2012.00177

26. de Lanerolle NC, Bandak F, Kang D, Li AY, Du F, Swauger P, Parks S, Ling G, Kim JH (2011) Characteristics of an explosive blast-induced brain injury in an experimental model. J Neuropathol Exp Neurol 70:1046-1057

27. Dickerson MR, Bailey ZS, Murphy SF, Urban MJ, VandeVord PJ (2020) Glial activation in the thalamus contributes to vestibulomotor deficits following blast-induced neurotrauma. Front Neurol 11:618. https://doi. org/10.3389/fneur.2020.00618

28. Dorr A, Sled JG, Kabani N (2007) Three-dimensional cerebral vasculature of the CBA mouse brain: a magnetic resonance imaging and micro computed tomography study. Neuroimage 35:1409-1423. https://doi. org/10.1016/j.neuroimage.2006.12.040

29. Drake CT, ladecola C (2007) The role of neuronal signaling in controlling cerebral blood flow. Brain Lang 102:141-152. https://doi.org/10.1016/j. bandl.2006.08.002

30. Elder GA, Dorr NP, De Gasperi R, Gama Sosa MA, Shaughness MC, Maudlin-Jeronimo E, Hall AA, McCarron RM, Ahlers ST (2012) Blast exposure induces post-traumatic stress disorder-related traits in a rat model of mild traumatic brain injury. J Neurotrauma 29:2564-2575. https://doi org/10.1089/neu.2012.2510

31. Elder GA, Ehrlich ME, Gandy S (2019) Relationship of traumatic brain injury to chronic mental health problems and dementia in military veterans. Neurosci Lett 707:134294. https://doi.org/10.1016/j.neulet. 2019.134294

32. Elder GA, Gama Sosa MA, De Gasperi R, Stone JR, Dickstein DL, Haghighi F, Hof PR, Ahlers ST (2015) Vascular and inflammatory factors in the pathophysiology of blast-induced brain injury. Front Neurol 6:48. https://doi.org/10.3389/fneur.2015.00048

33. Elder GA, Stone JR, Ahlers ST (2014) Effects of low-level blast exposure on the nervous system: is there really a controversy? Front Neurol 5:269. https://doi.org/10.3389/fneur.2014.00269

34. Filosa JA, Bonev AD, Nelson MT (2004) Calcium dynamics in cortical astrocytes and arterioles during neurovascular coupling. Circ Res 95:e73-81. https://doi.org/10.1161/01.RES.0000148636.60732.2e

35. Filosa JA, Bonev AD, Straub SV, Meredith AL, Wilkerson MK, Aldrich RW, Nelson MT (2006) Local potassium signaling couples neuronal activity to vasodilation in the brain. Nat Neurosci 9:1397-1403. https://doi.org/ $10.1038 / \mathrm{nn} 1779$

36. Filosa JA, Iddings JA (2013) Astrocyte regulation of cerebral vascular tone. Am J Physiol Heart Circ Physiol 305:H609-619. https://doi.org/10. 1152/ajpheart.00359.2013

37. Franciosi S, De Gasperi R, Dickstein DL, English DF, Rocher AB, Janssen WG, Christoffel D, Sosa MA, Hof PR, Buxbaum JD et al (2007) Pepsin pretreatment allows collagen IV immunostaining of blood vessels in adult mouse brain. J Neurosci Methods 163:76-82

38. Gama Sosa MA, De Gasperi R, Janssen PL, Yuk FJ, Anazodo PC, Pricop PE, Paulino AJ, Wicinski B, Shaughness MC, Maudlin-Jeronimo E et al (2014) Selective vulnerability of the cerebral vasculature to blast injury in a rat model of mild traumatic brain injury. Acta Neuropathol Commun 2:67. https://doi.org/10.1186/2051-5960-2-67

39. Gama Sosa MA, De Gasperi R, Paulino AJ, Pricop PE, Shaughness MC, Maudlin-Jeronimo E, Hall AA, Janssen WG, Yuk FJ, Dorr NP et al (2013) Blast overpressure induces shear-related injuries in the brain of rats exposed to a mild traumatic brain injury. Acta Neuropathol Commun 1:51. https://doi.org/10.1186/2051-5960-1-51

40. Gama Sosa MA, De Gasperi R, Perez Garcia GS, Perez GM, Searcy C, Vargas D, Spencer A, Janssen PL, Tschiffely AE, McCarron RM et al (2019) Low-level blast exposure disrupts gliovascular and neurovascular connections and induces a chronic vascular pathology in rat brain. Acta Neuropathol Commun 7:6. https://doi.org/10.1186/s40478-018-0647-5

41. Gama Sosa MA, De Gasperi R, Perez Garcia GS, Sosa H, Searcy C, Vargas D, Janssen PL, Perez GM, Tschiffely AE, Janssen WG et al (2017) Lack of chronic neuroinflammation in the absence of focal hemorrhage in a rat model of low-energy blast-induced TBI. Acta Neuropathol Commun 5:80. https://doi.org/10.1186/s40478-017-0483-z

42. Gama Sosa MA, Gasperi RD, Rocher AB, Wang AC, Janssen WG, Flores T, Perez GM, Schmeidler J, Dickstein DL, Hof PR et al (2010) Age-related vascular pathology in transgenic mice expressing presenilin 1-associated familial Alzheimer's disease mutations. Am J Pathol 176:353-368. https://doi.org/10.2353/ajpath.2010.090482

43. Goodrich JA, Kim JH, Situ R, Taylor W, Westmoreland T, Du F, Parks S, Ling G, Hwang JY, Rapuano A et al (2016) Neuronal and glial changes in the brain resulting from explosive blast in an experimental 
model. Acta Neuropathol Commun 4:124. https://doi.org/10.1186/ s40478-016-0395-3

44. Grebe A, Latz E (2013) Cholesterol crystals and inflammation. Curr Rheumatol Rep 15:313. https://doi.org/10.1007/s11926-012-0313-z

45. Hald ES, Alford PW (2014) Smooth muscle phenotype switching in blast traumatic brain injury-induced cerebral vasospasm. Transl Stroke Res 5:385-393. https://doi.org/10.1007/s12975-013-0300-3

46. Halder SK, Milner R (2019) A critical role for microglia in maintaining vascular integrity in the hypoxic spinal cord. Proc Natl Acad Sci U S A 116:26029-26037. https://doi.org/10.1073/pnas.1912178116

47. Hall CN, Reynell C, Gesslein B, Hamilton NB, Mishra A, Sutherland BA, O'Farrell FM, Buchan AM, Lauritzen M, Attwell D (2014) Capillary pericytes regulate cerebral blood flow in health and disease. Nature 508:55-60. https://doi.org/10.1038/nature13165

48. Hamel E (1985) (2006) Perivascular nerves and the regulation of cerebrovascular tone. J Appl Physiol 100:1059-1064. https://doi.org/10. 1152/japplphysiol.00954.2005

49. Hamilton NB, Attwell D, Hall CN (2010) Pericyte-mediated regulation of capillary diameter: a component of neurovascular coupling in health and disease. Front Neuroenergetics. https://doi.org/10.3389/fnene. 2010.00005

50. Han X, Chai Z, Ping X, Song LJ, Ma C, Ruan Y, Jin X (2020) In vivo twophoton imaging reveals acute cerebral vascular spasm and microthrombosis after mild traumatic brain injury in mice. Front Neurosci 14:210. https://doi.org/10.3389/fnins.2020.00210

51. Hao L, Du M, Lopez-Campistrous A, Fernandez-Patron C (2004) Agonistinduced activation of matrix metalloproteinase-7 promotes vasoconstriction through the epidermal growth factor-receptor pathway. Circ Res 94:68-76. https://doi.org/10.1161/01.RES.0000109413.57726.91

52. Haruwaka K, Ikegami A, Tachibana Y, Ohno N, Konishi H, Hashimoto A, Matsumoto M, Kato D, Ono R, Kiyama H et al (2019) Dual microglia effects on blood brain barrier permeability induced by systemic inflammation. Nat Commun 10:5816. https://doi.org/10.1038/ s41467-019-13812-z

53. Haurani MJ, Pagano PJ (2007) Adventitial fibroblast reactive oxygen species as autacrine and paracrine mediators of remodeling: bellwether for vascular disease? Cardiovasc Res 75:679-689. https://doi.org/10.1016/j. cardiores.2007.06.016

54. Hernandez A, Tan C, Plattner F, Logsdon AF, Pozo K, Yousuf MA, Singh T, Turner RC, Luke-Wold BP, Huber JD et al (2018) Exposure to mild blast forces induces neuropathological effects, neurophysiological deficits and biochemical changes. Mol Brain 11:64. https://doi.org/10.1186/ s13041-018-0408-1

55. Hicks RR, Baldwin SA, Scheff SW (1997) Serum extravasation and cytoskeletal alterations following traumatic brain injury in rats. Comparison of lateral fluid percussion and cortical impact models. Mol Chem Neuropathol 32:1-16. https://doi.org/10.1007/BF02815164

56. Hladky SB, Barrand MA (2018) Elimination of substances from the brain parenchyma: efflux via perivascular pathways and via the blood-brain barrier. Fluids Barriers CNS 15:30. https://doi.org/10.1186/ s12987-018-0113-6

57. Hoogland IC, Houbolt C, van Westerloo DJ, van Gool WA, van de Beek D (2015) Systemic inflammation and microglial activation: systematic review of animal experiments. J Neuroinflammation 12:114. https://doi. org/10.1186/s12974-015-0332-6

58. Hotta H (2016) Neurogenic control of parenchymal arterioles in the cerebral cortex. Prog Brain Res 225:3-39. https://doi.org/10.1016/bs.pbr. 2016.03.001

59. Howarth C (2014) The contribution of astrocytes to the regulation of cerebral blood flow. Front Neurosci 8:103. https://doi.org/10.3389/fnins. 2014.00103

60. ladecola C (2017) The neurovascular unit coming of age: a journey through neurovascular coupling in health and disease. Neuron 96:17-42. https://doi.org/10.1016/j.neuron.2017.07.030

61. Jessen NA, Munk AS, Lundgaard I, Nedergaard M (2015) The glymphatic system: a beginner's guide. Neurochem Res 40:2583-2599. https://doi. org/10.1007/s11064-015-1581-6

62. Johnson C, Galis ZS (2004) Matrix metalloproteinase-2 and -9 differentially regulate smooth muscle cell migration and cell-mediated collagen organization. Arterioscler Thromb Vasc Biol 24:54-60. https:// doi.org/10.1161/01.ATV.0000100402.69997.C3
63. Kabu S, Jaffer H, Petro M, Dudzinski D, Stewart D, Courtney A, Courtney M, Labhasetwar V (2015) Blast-associated shock waves result in increased brain vascular leakage and elevated ROS levels in a rat model of traumatic brain injury. PLoS ONE 10:e0127971. https://doi.org/10 1371/journal.pone.0127971

64. Kacza J, Seeger J (1997) Transcellular labelling of activated retinal microglia following transection of the optic nerve. Inflamm Res 46:430-433. https://doi.org/10.1007/s000110050219

65. Kallakuri S, Desai A, Feng K, Tummala S, Saif T, Chen C, Zhang L, Cavanaugh JM, King AI (2017) Neuronal injury and glial changes are hallmarks of open field blast exposure in swine frontal lobe. PLOS ONE 12:e0169239. https://doi.org/10.1371/journal.pone.0169239

66. Kawoos U, Abutarboush R, Gu M, Chen Y, Statz JK, Goodrich SY, Ahlers ST (2021) Blast-induced temporal alterations in blood-brain barrier properties in a rodent model. Sci Rep 11:5906. https://doi.org/10.1038/ s41598-021-84730-8

67. Kawoos U, Gu M, Lankasky J, McCarron RM, Chavko M (2016) Effects of exposure to blast overpressure on intracranial pressure and blood-brain barrier permeability in a rat model. PLoS ONE 11:e0167510. https://doi. org/10.1371/journal.pone.0167510

68. Kedarasetti RT, Drew PJ, Costanzo F (2020) Arterial pulsations drive oscillatory flow of CSF but not directional pumping. Sci Rep 10:10102. https://doi.org/10.1038/s41598-020-66887-w

69. Kettenmann H, Hanisch UK, Noda M, Verkhratsky A (2011) Physiology of microglia. Physiol Rev 91:461-553. https://doi.org/10.1152/physrev. 00011.2010

70. Kisler K, Nelson AR, Montagne A, Zlokovic BV (2017) Cerebral blood flow regulation and neurovascular dysfunction in Alzheimer disease. Nat Rev Neurosci 18:419-434. https://doi.org/10.1038/nrn.2017.48

71. Kisler K, Nelson AR, Rege SV, Ramanathan A, Wang Y, Ahuja A, Lazic D, Tsai PS, Zhao Z, Zhou Y et al (2017) Pericyte degeneration leads to neurovascular uncoupling and limits oxygen supply to brain. Nat Neurosci 20:406-416. https://doi.org/10.1038/nn.4489

72. Kofuji P, Araque A (2021) Astrocytes and behavior. Annu Rev Neurosci. https://doi.org/10.1146/annurev-neuro-101920-112225

73. Kreutzberg GW (1996) Microglia: a sensor for pathological events in the CNS. Trends Neurosci 19:312-318

74. KulikT, Kusano Y, Aronhime S, Sandler AL, Winn HR (2008) Regulation of cerebral vasculature in normal and ischemic brain. Neuropharmacology 55:281-288. https://doi.org/10.1016/..neuropharm.2008.04.017

75. Kuriakose M, Rama Rao KV, Younger D, Chandra N (2018) Temporal and spatial effects of blast overpressure on blood-brain barrier permeability in traumatic brain injury. Sci Rep 8:8681. https://doi.org/10.1038/ s41598-018-26813-7

76. Lannes N, Eppler E, Etemad S, Yotovski P, Filgueira L (2017) Microglia at center stage: a comprehensive review about the versatile and unique residential macrophages of the central nervous system. Oncotarget 8:114393-114413. https://doi.org/10.18632/oncotarget.23106

77. Lee HS, Ghetti A, Pinto-Duarte A, Wang X, Dziewczapolski G, Galimi F, Huitron-Resendiz S, Pina-Crespo JC, Roberts AJ, Verma IM et al (2014) Astrocytes contribute to gamma oscillations and recognition memory. Proc Natl Acad Sci U S A 111:E3343-3352. https://doi.org/10.1073/pnas. 1410893111

78. Li G, Chen SJ, Oparil S, Chen YF, Thompson JA (2000) Direct in vivo evidence demonstrating neointimal migration of adventitial fibroblasts after balloon injury of rat carotid arteries. Circulation 101:1362-1365. https://doi.org/10.1161/01.cir.101.12.1362

79. Li H, Xu H, Sun B (2012) Lipopolysaccharide regulates MMP-9 expression through TLR4/NF-kappaB signaling in human arterial smooth muscle cells. Mol Med Rep 6:774-778. https://doi.org/10.3892/mmr. 2012.1010

80. Lieberman AP, Swanson JA (2018) High cholesterol at the heart of phagolysosomal damage. Cell Metab 27:487-488. https://doi.org/10. 1016/j.cmet.2018.02.015

81. Logsdon AF, Meabon JS, Cline MM, Bullock KM, Raskind MA, Peskind ER, Banks WA, Cook DG (2018) Blast exposure elicits blood-brain barrier disruption and repair mediated by tight junction integrity and nitric oxide dependent processes. Sci Rep 8:11344. https://doi.org/10.1038/ s41598-018-29341-6

82. Logsdon AF, Schindler AG, Meabon JS, Yagi M, Herbert MJ, Banks WA, Raskind MA, Marshall DA, Keene CD, Perl DP et al (2020) Nitric oxide 
synthase mediates cerebellar dysfunction in mice exposed to repetitive blast-induced mild traumatic brain injury. Sci Rep 10:9420. https://doi. org/10.1038/s41598-020-66113-7

83. Mac Donald CL, Barber J, Patterson J, Johnson AM, Dikmen S, Fann $J R$, Temkin N (2019) Association between 5-year clinical outcome in patients with nonmedically evacuated mild blast traumatic brain injury and clinical measures collected within 7 days postinjury in combat. JAMA Netw Open 2:e186676. https://doi.org/10.1001/jamanetwor kopen.2018.6676

84. Mackie AR, Losordo DW (2011) CD34-positive stem cells: in the treatment of heart and vascular disease in human beings. Tex Heart Inst J 38:474-485

85. MacVicar BA, Newman EA (2015) Astrocyte regulation of blood flow in the brain. Cold Spring Harb Perspect Biol. https://doi.org/10.1101/ cshperspect.a020388

86. Marina N, Christie IN, Korsak A, Doronin M, Brazhe A, Hosford PS, Wells JA, Sheikhbahaei S, Humoud I, Paton JFR et al (2020) Astrocytes monitor cerebral perfusion and control systemic circulation to maintain brain blood flow. Nat Commun 11:131. https://doi.org/10.1038/ s41467-019-13956-y

87. Martinez-Lemus LA (2012) The dynamic structure of arterioles. Basic Clin Pharmacol Toxicol 110:5-11. https://doi.org/10.1111/j.1742-7843. 2011.00813.x

88. Martinez-Lemus LA, Zhao G, Galinanes EL, Boone M (2011) Inward remodeling of resistance arteries requires reactive oxygen speciesdependent activation of matrix metalloproteinases. Am J Physiol Heart Circ Physiol 300:H2005-2015. https://doi.org/10.1152/ajpheart.01066. 2010

89. Mishra A, Reynolds JP, Chen Y, Gourine AV, Rusakov DA, Attwell D (2016) Astrocytes mediate neurovascular signaling to capillary pericytes but not to arterioles. Nat Neurosci 19:1619-1627. https://doi.org/10.1038/ nn. 4428

90. Mulligan SJ, MacVicar BA (2004) Calcium transients in astrocyte endfeet cause cerebrovascular constrictions. Nature 431:195-199. https://doi. org/10.1038/nature02827

91. Naganawa S, Taoka T (2020) The glymphatic system: a review of the challenges in visualizing its structure and function with MR imaging Magn Reson Med Sci. https://doi.org/10.2463/mrms.rev.2020-0122

92. Nedergaard M, Ransom B, Goldman SA (2003) New roles for astrocytes: redefining the functional architecture of the brain. Trends Neurosci 26:523-530. https://doi.org/10.1016/j.tins.2003.08.008

93. Newby AC (2006) Matrix metalloproteinases regulate migration, proliferation, and death of vascular smooth muscle cells by degrading matrix and non-matrix substrates. Cardiovasc Res 69:614-624. https://doi.org/ 10.1016/j.cardiores.2005.08.002

94. Odenbach J, Wang X, Cooper S, Chow FL, Oka T, Lopaschuk G, Kassiri Z, Fernandez-Patron C (2011) MMP-2 mediates angiotensin II-induced hypertension under the transcriptional control of MMP-7 and TACE. Hypertension 57:123-130. https://doi.org/10.1161/HYPERTENSIONAHA. 110.159525

95. Omri S, Behar-Cohen F, de Kozak Y, Sennlaub F, Verissimo LM, Jonet L, Savoldelli M, Omri B, Crisanti P (2011) Microglia/macrophages migrate through retinal epithelium barrier by a transcellular route in diabetic retinopathy: role of PKCzeta in the Goto Kakizaki rat model. Am J Pathol 179:942-953. https://doi.org/10.1016/j.ajpath.2011.04.018

96. Ota Y, Zanetti AT, Hallock RM (2013) The role of astrocytes in the regulation of synaptic plasticity and memory formation. Neural Plast 2013:185463. https://doi.org/10.1155/2013/185463

97. Paxinos $G$, Watson C (2007) The rat brain in stereotaxic coordinates. Academic Press, London

98. Pekny M, Pekna M (2014) Astrocyte reactivity and reactive astrogliosis: costs and benefits. Physiol Rev 94:1077-1098. https://doi.org/10.1152/ physrev.00041.2013

99. Perez Garcia G, Perez GM, De Gasperi R, Gama Sosa MA, Otero-Pagan A, Pryor D, Abutarboush R, Kawoos U, Hof PR, Cook DG et al (2021) Progressive cognitive and post-traumatic stress disorder-related behavioral traits in rats exposed to repetitive low-level blast. J Neurotrauma. https://doi.org/10.1089/neu.2020.7398

100. Perez-Garcia G, Gama Sosa MA, De Gasperi R, Lashof-Sullivan M, Maudlin-Jeronimo E, Stone JR, Haghighi F, Ahlers ST, Elder GA (2018) Chronic post-traumatic stress disorder-related traits in a rat model of low-level blast exposure. Behav Brain Res 340:117-125. https://doi.org/ 10.1016/j.bbr.2016.09.061

101. Perez-Garcia G, Gama Sosa MA, De Gasperi R, Lashof-Sullivan M, Maudlin-Jeronimo E, Stone JR, Haghighi F, Ahlers ST, Elder GA (2016) Exposure to a predator scent induces chronic behavioral changes in rats previously exposed to low-level blast: implications for the relationship of blast-related TBI to PTSD. Front Neurol 7:176. https://doi.org/10. 3389/fneur.2016.00176

102. Petrie EC, Cross DJ, Yarnykh VL, Richards T, Martin NM, Pagulayan K, Hoff D, Hart K, Mayer C, Tarabochia M et al (2014) Neuroimaging, behavioral, and psychological sequelae of repetitive combined blast/impact mild traumatic brain injury in Iraq and Afghanistan war veterans. J Neurotrauma 31:425-436. https://doi.org/10.1089/neu.2013.2952

103. Petronilli V, Penzo D, Scorrano L, Bernardi P, Di Lisa F (2001) The mitochondrial permeability transition, release of cytochrome $c$ and cell death. Correlation with the duration of pore openings in situ. J Biol Chem 276:12030-12034. https://doi.org/10.1074/jbc.M010604200

104. Piantino J, Schwartz DL, Luther M, Newgard C, Silbert L, Raskind M, Pagulayan K, Kleinhans N, lliff J, Peskind E (2021) Link between mild traumatic brain injury, poor sleep, and magnetic resonance imaging: visible perivascular spaces in veterans. J Neurotrauma. https://doi.org/ 10.1089/neu.2020.7447

105. Plaut A (1957) The notched nucleus of the fat cell (Unna's lochkern). J Mt Sinai Hosp N Y 24:1112-1120

106. Price L, Wilson C, Grant G (2016) Blood-brain barrier pathophysiology following traumatic brain injury. In: Laskowitz D, Grant G (eds) Translational research in traumatic brain injury. CRC Press, Boca Raton

107. Prinz M, Masuda T, Wheeler MA, Quintana FJ (2021) Microglia and central nervous system-associated macrophages-from origin to disease modulation. Annu Rev Immunol. https://doi.org/10.1146/annurevimmunol-093019-110159

108. Prockop DJ, Kivirikko KI (1995) Collagens: molecular biology, diseases, and potentials for therapy. Annu Rev Biochem 64:403-434. https://doi. org/10.1146/annurev.bi.64.070195.002155

109. Puro DG (2007) Physiology and pathobiology of the pericyte-containing retinal microvasculature: new developments. Microcirculation 14:1-10. https://doi.org/10.1080/10739680601072099

110. Rajamaki K, Lappalainen J, Oorni K, Valimaki E, Matikainen S, Kovanen PT, Eklund KK (2010) Cholesterol crystals activate the NLRP3 inflammasome in human macrophages: a novel link between cholesterol metabolism and inflammation. PLoS ONE 5:e1 1765. https://doi.org/10. 1371/journal.pone.0011765

111. Rashid K, Akhtar-Schaefer I, Langmann T (2019) Microglia in retinal degeneration. Front Immunol 10:1975. https://doi.org/10.3389/fimmu. 2019.01975

112. Robinson ME, Clark DC, Milberg WP, McGlinchey RE, Salat DH (2017) Characterization of differences in functional connectivity associated with close-range blast exposure. J Neurotrauma 34:S53-S61. https:// doi.org/10.1089/neu.2016.4709

113. Rodriguez UA, Zeng Y, Deyo D, Parsley MA, Hawkins BE, Prough DS, DeWitt DS (2018) Effects of mild blast traumatic brain injury on cerebral vascular, histopathological, and behavioral outcomes in rats. J Neurotrauma 35:375-392. https://doi.org/10.1089/neu.2017.5256

114. Rodriguez UA, Zeng Y, Parsley MA, Hawkins BE, Prough DS, DeWitt DS (2019) Effects of blast-induced neurotrauma on pressurized rodent middle cerebral arteries. J Vis Exp. https://doi.org/10.3791/58792

115. Rosenblatt AS, Li R, Fortier C, Liu X, Fonda JR, Villalon A, McGlinchey RE, Jorge RE (2019) Latent factor structure of PTSD symptoms in veterans with a history of mild traumatic brain injury and close-range blast exposure. Psychol Trauma 11:442-450. https://doi.org/10.1037/tra0000399

116. Rubio JE, Skotak M, Alay E, Sundaramurthy A, Subramaniam DR, Kote VB, Yeoh S, Monson K, Chandra N, Unnikrishnan G et al (2020) Does blast exposure to the torso cause a blood surge to the brain? Front Bioeng Biotechnol 8:573647. https://doi.org/10.3389/fbioe.2020.573647

117. Rubio JE, Unnikrishnan G, Sajja V, Van Albert S, Rossetti F, Skotak M, Alay E, Sundaramurthy A, Subramaniam DR, Long JB et al (2021) Investigation of the direct and indirect mechanisms of primary blast insult to the brain. Sci Rep 11:16040. https://doi.org/10.1038/s41598-021-95003-9

118. Schetters STT, Gomez-Nicola D, Garcia-Vallejo JJ, Van Kooyk Y (2017) Neuroinflammation: microglia and T cells get ready to tango. Front Immunol 8:1905. https://doi.org/10.3389/fimmu.2017.01905 
119. Shapira Y, Setton D, Artru AA, Shohami E (1993) Blood-brain barrier permeability, cerebral edema, and neurologic function after closed head injury in rats. Anesth Analg 77:141-148. https://doi.org/10.1213/00000 539-199307000-00028

120. Sheng JG, Mrak RE, Griffin WS (1997) Neuritic plaque evolution in Alzheimer's disease is accompanied by transition of activated microglia from primed to enlarged to phagocytic forms. Acta Neuropathol 94:1-5

121. Shepro D, Morel NM (1993) Pericyte physiology. FASEB J 7:1031-1038

122. Shively SB, Horkayne-Szakaly I, Jones RV, Kelly JP, Armstrong RC, Perl DP (2016) Characterisation of interface astroglial scarring in the human brain after blast exposure: a post-mortem case series. Lancet Neurol 15:944-953. https://doi.org/10.1016/S1474-4422(16)30057-6

123. Skotak M, Townsend MT, Ramarao KV, Chandra N (2019) A Comprehensive review of experimental rodent models of repeated blast TBI. Front Neurol 10:1015. https://doi.org/10.3389/fneur.2019.01015

124. Soltys Z, Ziaja M, Pawlinski R, Setkowicz Z, Janeczko K (2001) Morphology of reactive microglia in the injured cerebral cortex. Fractal analysis and complementary quantitative methods. J Neurosci Res 63:90-97. https://doi.org/10.1002/1097-4547(20010101)63:1\%3c90::AID-JNR1 1\% 3e3.0.CO;2-9

125. Squire LR (2013) Fundamental neuroscience. Elsevier, Amsterdam

126. St-Pierre MK, Simoncicova E, Bogi E, Tremblay ME (2020) Shedding light on the dark side of the microglia. ASN Neuro 12:1759091420925335. https://doi.org/10.1177/1759091420925335

127. Steffensen LB, Rasmussen LM (2018) A role for collagen type IV in cardiovascular disease? Am J Physiol Heart Circ Physiol 315:H610-H625. https://doi.org/10.1152/ajpheart.00070.2018

128. Stence N, Waite M, Dailey ME (2001) Dynamics of microglial activation: a confocal time-lapse analysis in hippocampal slices. Glia 33:256-266

129. Stenmark KR, Yeager ME, El Kasmi KC, Nozik-Grayck E, Gerasimovskaya EV, Li M, Riddle SR, Frid MG (2013) The adventitia: essential regulator of vascular wall structure and function. Annu Rev Physiol 75:23-47. https://doi.org/10.1146/annurev-physiol-030212-183802

130. Stone JR, Avants BB, Tustison NJ, Wassermann EM, Gill J, Polejaeva E, Dell KC, Carr W, Yarnell AM, LoPresti ML et al (2020) Functional and structural neuroimaging correlates of repetitive low-level blast exposure in career breachers. J Neurotrauma 37:2468-2481. https://doi.org/10.1089/neu. 2020.7141

131. Sullivan DR (2019) A cerebrovascular hypothesis of neurodegeneration in mTBI. J Head Trauma Rehabil 34:E18-E27. https://doi.org/10.1097/ HTR.0000000000000449

132. Sullivan DR, Miller MW, Wolf EJ, Logue MW, Robinson ME, Fortier CB, Fonda JR, Wang DJ, Milberg WP, McGlinchey RE et al (2020) Cerebral perfusion is associated with blast exposure in military personnel without moderate or severe TBI. J Cereb Blood Flow Metab. https://doi.org/ 10.1177/0271678X20935190

133. Sullivan DR, Miller MW, Wolf EJ, Logue MW, Robinson ME, Fortier CB, Fonda JR, Wang DJ, Milberg WP, McGlinchey RE et al (2021) Cerebral perfusion is associated with blast exposure in military personnel without moderate or severe TBI. J Cereb Blood Flow Metab 41:886-900. https://doi.org/10.1177/0271678X20935190

134. Sullivan EV (2012) War-related PTSD, blast injury, and anosognosia. Neuropsychol Rev 22:1-2. https://doi.org/10.1007/s11065-012-9188-z

135. Suzuki A, Stern SA, Bozdagi O, Huntley GW, Walker RH, Magistretti PJ, Alberini CM (2011) Astrocyte-neuron lactate transport is required for long-term memory formation. Cell 144:810-823. https://doi.org/10. 1016/j.cell.2011.02.018

136. Szentistvanyi I, Patlak CS, Ellis RA, Cserr HF (1984) Drainage of interstitial fluid from different regions of rat brain. Am J Physiol 246:F835-844. https://doi.org/10.1152/ajprenal.1984.246.6.F835

137. Thanos S, Pavlidis C, Mey J, Thiel HJ (1992) Specific transcellular staining of microglia in the adult rat after traumatic degeneration of carbocyanine-filled retinal ganglion cells. Exp Eye Res 55:101-117. https://doi. org/10.1016/0014-4835(92)90098-d

138. Toklu HZ, Muller-Delp J, Yang Z, Oktay S, Sakarya Y, Strang K, Ghosh P, Delp MD, Scarpace PJ, Wang KK et al (2015) The functional and structural changes in the basilar artery due to overpressure blast injury. J Cereb Blood Flow Metab 35:1950-1956. https://doi.org/10.1038/jcbfm. 2015.151

139. Torres-Platas SG, Comeau S, Rachalski A, Bo GD, Cruceanu C, Turecki G, Giros B, Mechawar N (2014) Morphometric characterization of microglial phenotypes in human cerebral cortex. J Neuroinflammation 11:12. https://doi.org/10.1186/1742-2094-11-12

140. Troyanskaya M, Pastorek NJ, Scheibel RS, Petersen NJ, McCulloch K, Wilde EA, Henson HK, Levin HS (2015) Combat exposure, PTSD symptoms, and cognition following blast-related traumatic brain injury in OEF/OIF/OND service members and Veterans. Mil Med 180:285-289. https://doi.org/10.7205/MILMED-D-14-00256

141. Trudeau DL, Anderson J, Hansen LM, Shagalov DN, Schmoller J, Nugent S, Barton S (1998) Findings of mild traumatic brain injury in combat veterans with PTSD and a history of blast concussion. J Neuropsychiatry Clin Neurosci 10:308-313. https://doi.org/10.1176/jnp.10.3.308

142. Ueno M, Chiba Y, Murakami R, Matsumoto K, Fujihara R, Uemura N, Yanase K, Kamada M (2019) Disturbance of intracerebral fluid clearance and blood-brain barrier in vascular cognitive impairment. Int J Mol Sci. https://doi.org/10.3390/ijms20102600

143. Varnum MM, Ikezu T (2012) The classification of microglial activation phenotypes on neurodegeneration and regeneration in Alzheimer's disease brain. Arch Immunol Ther Exp (Warsz) 60:251-266. https://doi. org/10.1007/s00005-012-0181-2

144. Vieillard-Baron A, Frisdal E, Raffestin B, Baker AH, Eddahibi S, Adnot S, D'Ortho MP (2003) Inhibition of matrix metalloproteinases by lung TIMP-1 gene transfer limits monocrotaline-induced pulmonary vascular remodeling in rats. Hum Gene Ther 14:861-869. https://doi.org/10. 1089/104303403765701150

145. Zagorchev L, Oses P, Zhuang ZW, Moodie K, Mulligan-Kehoe MJ, Simons M, Couffinhal T (2010) Micro computed tomography for vascular exploration. J Angiogenes Res 2:7. https://doi.org/10.1186/2040-2384-2-7

146. Zonta M, Angulo MC, Gobbo S, Rosengarten B, Hossmann KA, Pozzan T, Carmignoto G (2003) Neuron-to-astrocyte signaling is central to the dynamic control of brain microcirculation. Nat Neurosci 6:43-50. https://doi.org/10.1038/nn980

\section{Publisher's Note}

Springer Nature remains neutral with regard to jurisdictional claims in published maps and institutional affiliations.

Ready to submit your research? Choose BMC and benefit from:

- fast, convenient online submission

- thorough peer review by experienced researchers in your field

- rapid publication on acceptance

- support for research data, including large and complex data types

- gold Open Access which fosters wider collaboration and increased citations

- maximum visibility for your research: over 100M website views per year

At BMC, research is always in progress.

Learn more biomedcentral.com/submissions 